\title{
Quantized Transform-Domain Motion Estimation for SP-Frame Coding in Viewpoint Switching of Multiview Video
}

\author{
Ki-Kit Lai, Yui-Lam Chan, Member, IEEE, and Wan-Chi Siu, Senior Member, IEEE
}

\begin{abstract}
The brand-new SP-frame in $\mathbf{H} .264$ facilitates drift-free bitstream switching. Notwithstanding the guarantee of seamless switching, the cost is the bulky size of secondary SP-frames. This induces a significant amount of additional space or bandwidth for storage or transmission. In this paper, our investigation reveals that the size of secondary SP-frames is more severe when the correlation between the two bitstreams becomes smaller. Examples include viewpoint switching in multiview video and bitstream switching in single-view video with complex motion. For this reason, a new motion estimation and compensation technique, which is operated in the quantizedtransform (QDCT) domain, is designed for coding secondary SP-frames. Our proposed work aims at keeping the secondary SP-frames as small as possible without affecting the size of primary SP-frames by incorporating QDCT-domain motion estimation and compensation in the secondary SP-frame coding. Simulation results show our proposed scheme overwhelmingly outperforms the conventional pixel-domain motion estimation technique. As a consequence, the size of secondary SP-frames can be reduced remarkably, especially in multiview video and single-view video with complex motion.
\end{abstract}

Index Terms-Bitstream switching, motion estimation, multiview video, QDCT domain, SP-frame, viewpoint switching.

\section{INTRODUCTION}

$\mathbf{H}$ .264 IS THE state-of-the-art video coding standard [1]-[3] developed by the ITU-T/ISO/IEC Joint Video Team consisting of experts from the ISO/IEC's Moving Picture Experts Group (MPEG) and the ITU-T's Video Coding Experts Group. H.264 has been shown to achieve a compression gain of 1.5 to 2.0 times over previous video coding standards such as H.263+ and MPEG-4 Part 2 [1]-[3]. In addition to achieving superior coding efficiency, the H.264 standard includes an extensive set of new features to provide more flexibility for applications to a wide variety of network environments. The new SP-frame [4], [5] is one of these fea-

Manuscript received April 14, 2008; revised August 16, 2008, December 29, 2008, and May 8, 2009. First version published November 3, 2009; current version published March 5, 2010. This work was supported in part by the Center for Signal Processing, Department of Electronic and Information Engineering, The Hong Kong Polytechnic University, and by the Research Grants Council of the Hong Kong Special Administrative Region, China, under Grant PolyU 5125/06E. The work of K.-K. Lai was supported by the research studentships provided by The Hong Kong Polytechnic University. This paper was recommended by Associate Editor Y.-S. Ho.

The authors are with the Center for Signal Processing, Department of Electronic and Information Engineering, The Hong Kong Polytechnic University, Hung Hom, Kowloon, Hong Kong (e-mail: kikit.lai@polyu.edu.hk; enylchan@polyu.edu.hk; enwcsiu@polyu.edu.hk).

Digital Object Identifier 10.1109/TCSVT.2009.2035835 tures. The motivation of introducing SP-frames is to facilitate efficient switching between video streams for multiple bitrate video (MBV) streaming to accommodate bandwidth variation. It is now part of the Extended Profile in the H.264 standard, which may be particularly useful for applications such as video streaming. This special SP-frame scheme is composed of primary and secondary SP-frames. They both exploit temporal redundancy with predictive coding, but use different reference frames. Although different reference frames are used, it still allows an identical reconstruction. This property allows the use of SP-frames to replace I-frames for drift-free switching between compressed bitstreams of different quality and bitrates. Unlike I-frames, primary SP-frames are making use of motion-compensated predictive coding. For that reason, the coding efficiency of a primary SP-frame is much better than that of an I-frame and is slightly worse than that of a Pframe [5]-[7]. Nevertheless, extra storage for secondary SPframes is inevitably required. In [6]-[8], some investigations were conducted to evaluate the trade-off between the coding performance of primary SP-frames and the storage cost of secondary SP-frames for MBV applications. It is found that a primary SP-frame with high quality results in a significantly high storage requirement for a secondary SP-frame.

Meanwhile, multiview video (MVV) service has been attracting more and more attention recently. Different from the traditional single-view video, MVV is a collection of multiple videos that are captured simultaneously from multiple cameras placed at different locations. These multiple videos enable different types of application scenarios depending on the adoption of coding techniques. On the one hand, the MPEG committee of the 3-D audio-visual activity [9]-[12] group has worked toward the standardization for multiview video coding (MVC) with exploiting both temporal and interview [13], [14] correlations by combining motion and interview prediction. The inter-view prediction supported in the MVC standard can increase coding efficiency for all views and it is well suited for free viewpoint video and 3-D video in which all views should be decoded in the 3-D display [10]. On the other hand, some multiview systems encode multiple videos independently (simulcast-coded) in order to facilitate a brand new viewing experience with a high degree of user interactivity. In the conventional single-view video, it only provides one view direction for an event at any time instance whereas users may want to watch the event from 


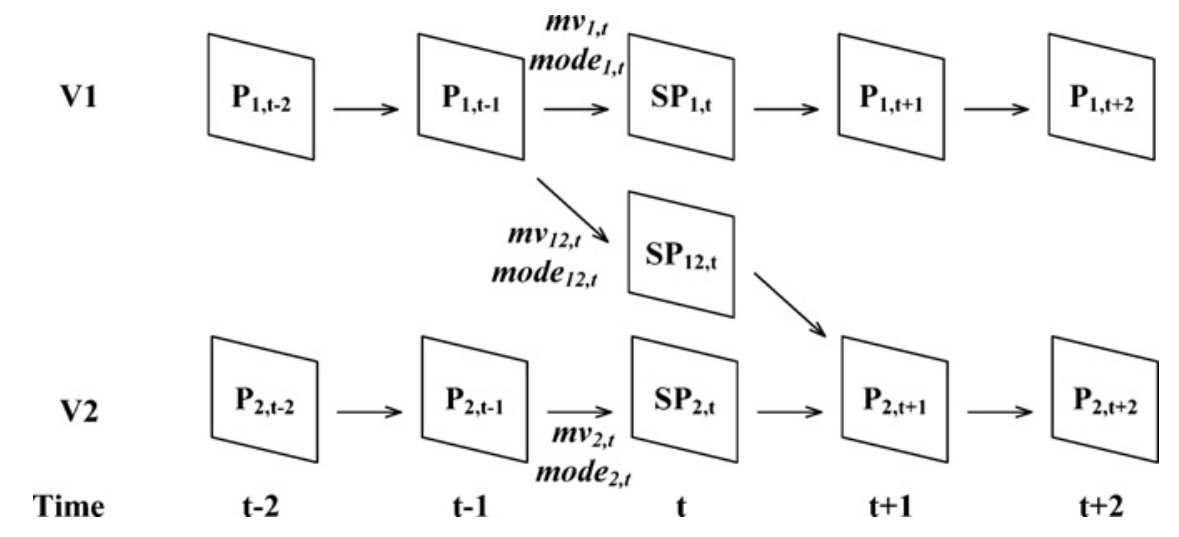

Fig. 1. Switching bitstream from V1 to V2 using SP-frames.

other directions. It means the users are very passive and can only watch the pre-selected video contents while there is no interactivity between the users and the content provider. With multiple videos, users can view a program with the freedom of view direction selection. Owing to its promising features, there are a large number of conceivable applications, including interactive advertisement, educational program, sport games, etc. Although the inter-view prediction can provide better coding efficiency in the MVC standard, multiple views should be transmitted for decoding a single view due to its complex view-dependency structure. It is not a practical way to adopt the inter-view prediction in this scenario where viewpoint switching occurs frequently [15]-[18]. In practical systems available in the market, it is desirable to develop a lowdelay viewpoint switching scheme which can switch among different views upon requests instead of transmitting all the views. These systems provide interactive functions for the client users in multiview video systems. For instance, an EyeVision system [19] was employed to shoot Super Bowl 2001 by using 30 cameras placed at different angles. Besides, in [17], [18], a new streaming system for multiview video was developed by Microsoft, which collects a number of video streams. These video streams are compressed independently (simulcast-coded) from control units. It provides interactive and reliable multiview video streaming based on server-client model. When views are simulcast-coded, this system can offer the interactive features such as viewpoint switching, frozen moment, and view sweeping. But, in these practical systems, switching between the bitstreams of different views is only allowed at I-frames, where the coding process does not rely on information from any previous frame, in order to avoid severe drifting problem. It is well-known that I-frames cost much more bits than P-frames. The interval between each I-frame in the bitstream of each view thus always keeps far apart. In this arrangement, switching cannot take place at any desired frame and this greatly affects the flexibility of the viewpoint switching capability. Alternatively, if I-frames are frequently inserted to support rapid switching between bitstreams of different viewpoints, the coding efficiency will be greatly reduced.

Therefore, the SP-frame encoding can be directly adopted for low-delay viewpoint switching in multiview streaming applications. Our investigation reveals that the bulky size of secondary SP-frames is more severe in MVV due to the presence of disparity between views resulting in reduction of correlations. It is also noted that the drastic increase in size of secondary SP-frames also happens in the scenario of bitstream switching in any scene of MBV with complex motion. Besides, for $\mathrm{MVV}$, an additional sequence of secondary SP-frames is required between each view pair. For example, the extra $N-1$ sequences of secondary SP-frames are stored in the MVV system with $N$ views. There is a great need for reducing the size of secondary SP-frames, which can alleviate the storage requirement of the server. In this paper, we propose a novel coding arrangement to reduce the size of secondary SPframes. We identify the problem of using the traditional pixeldomain motion estimation and compensation when secondary SP-frames are employed in viewpoint or bitstream switching for MVV and MBV. After the analysis, a novel SP-frame coding scheme using quantized-transform (QDCT) domain is proposed to reduce the size of secondary SP-frames. The proposed coding arrangement of secondary SP-frames will not affect the coding efficiency of primary SP-frames. It means that the playback quality of each single view without switching can be kept.

The rest of the paper is organized as follows. Section II gives a brief description of the conventional SP-frame encoding process. An in-depth study of the problem on applying the traditional pixel-domain motion estimation technique into a secondary SP-frame encoder is provided in Section III. Analysis of using QDCT-domain motion estimation is also covered in this section. After the detailed investigation, a novel secondary SP-frame encoder is proposed. In Section IV, experimental results are shown, which focus on the performance of our proposed scheme over the conventional secondary SPframe encoder in both MVV and MBV. Finally, Section V concludes this paper.

\section{ENCODING OF SP-FRAMES}

The purpose of this paper is to reduce the size of secondary SP-frames when they are adopted in switching compressed bitstreams with relatively low correlation. Examples include viewpoint switching in MVV and bitstream switching in MBV 


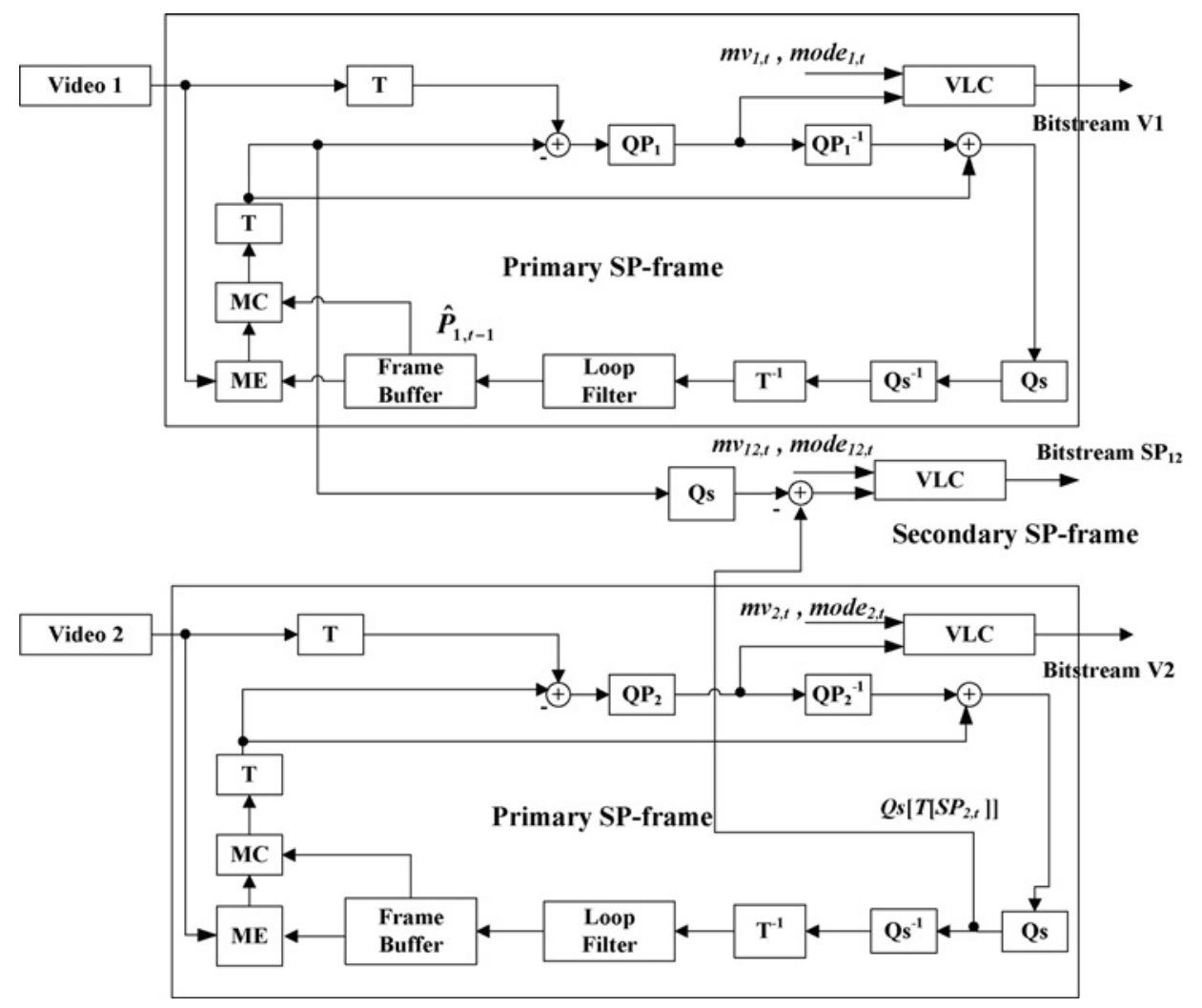

Fig. 2. Simplified encoding block diagram of primary and secondary SP-frames [4], [5].

with complex motion. For simplicity but without loss of generality, we focus our discussions on the case of viewpoint switching in MVV. The results can be easily extended to bitstream switching in MBV with complex motion and will also be given in Section IV.

Fig. 1 illustrates the idea of viewpoint switching in a multiview video using SP-frames. In this figure, two different views, which are captured by two cameras at the same time in the same scene, are encoded into two bitstreams (V1 and V2). V1 is an encoded bitstream in viewpoint 1 while V2 is in viewpoint 2 . Within each view, two primary SP-frames$S P_{1, t}$ and $S P_{2, t}$ are placed at frame $t$ (switching point). To allow seamless switching, a secondary SP-frame $\left(S P_{12, t}\right)$ is produced, which has the same reconstructed values as $S P_{2, t}$ even different reference frames are used. When switching from $\mathrm{V} 1$ to V2 is needed at frame $t, S P_{12, t}$ instead of $S P_{2, t}$ is transmitted. After decoding $S P_{12, t}$, the decoder can obtain exactly the same reconstructed values as normally $S P_{2, t}$ decoded at frame $t$. Therefore, it can continually decode $\mathrm{V} 2$ at frame $t+1$ seamlessly.

The way of encoding primary SP-frames is similar to that of encoding P-frames except additional quantization/dequantization steps with the quantization level $Q s$ are applied to the transform coefficients of the primary SP-frame $\left(S P_{2, t}\right.$ in Fig. 1), as shown in Fig. 2. Interested readers are encouraged to read [4], [5]. These extra steps ensure that the quantized-transform coefficients of $S P_{2, t}$ (denoted as $\left.Q s\left[T\left[S P_{2, t}\right]\right]\right)$ are divisible by $Q s$, which is used in the encoding process of the secondary SP-frame, $S P_{12, t}$.
For coding $S P_{12, t,}$ the reconstructed $P_{1, t-1}\left(\hat{P}_{1, t-1}\right)$ acts as the reference and its target is to reconstruct $Q s\left[T\left[S P_{2, t}\right]\right]$ perfectly. By using the reference frame $\hat{P}_{1, t-1}$, its prediction is first transformed and quantized using $Q s$ before generating the residue with $Q s\left[T\left[S P_{2, t}\right]\right]$. Both the prediction and $Q s\left[T\left[S P_{2, t}\right]\right]$ are thus synchronized to $Q s$ and there is no further quantization from this point, meaning that the decoder, with $\hat{P}_{1, t-1}, Q s$, and the residue available, can perfectly reconstruct $Q s\left[T\left[S P_{2, t}\right]\right]$.

Producing secondary SP-frames also involves the processes of motion estimation and compensation. In H.264, it supports motion estimation using different block sizes such as $16 \times 16$, $16 \times 8,8 \times 16,8 \times 8,8 \times 4,4 \times 8$, and $4 \times 4$ [2], [3]. To compute the coding modes and motion vectors for the secondary SP-frame, motion estimation is first performed for all modes and submodes independently by minimizing the Lagrangian cost function $J_{\text {motion }}$

$$
\begin{aligned}
& J_{\text {motion }}\left(m v_{12}, \lambda_{\text {motion }}\right) \\
& =S A D(s, r)+\lambda_{\text {motion }} \cdot R_{\text {motion }}\left(m v_{12}-p m v_{12}\right)
\end{aligned}
$$

where $m v_{12}$ is the motion vector used for prediction, $\lambda_{\text {motion }}$ is the Lagrangian multiplier for motion estimation, $R_{\text {motion }}\left(m v_{12}-p v_{12}\right)$ is the estimated number of bits for coding $m v_{12}$, and SAD is the sum of absolute differences between the original block $s$ and its reference block $r$ [2], [3].

After motion estimation for each mode, a rate-distortion (RD) optimization technique is used to obtain the best mode 


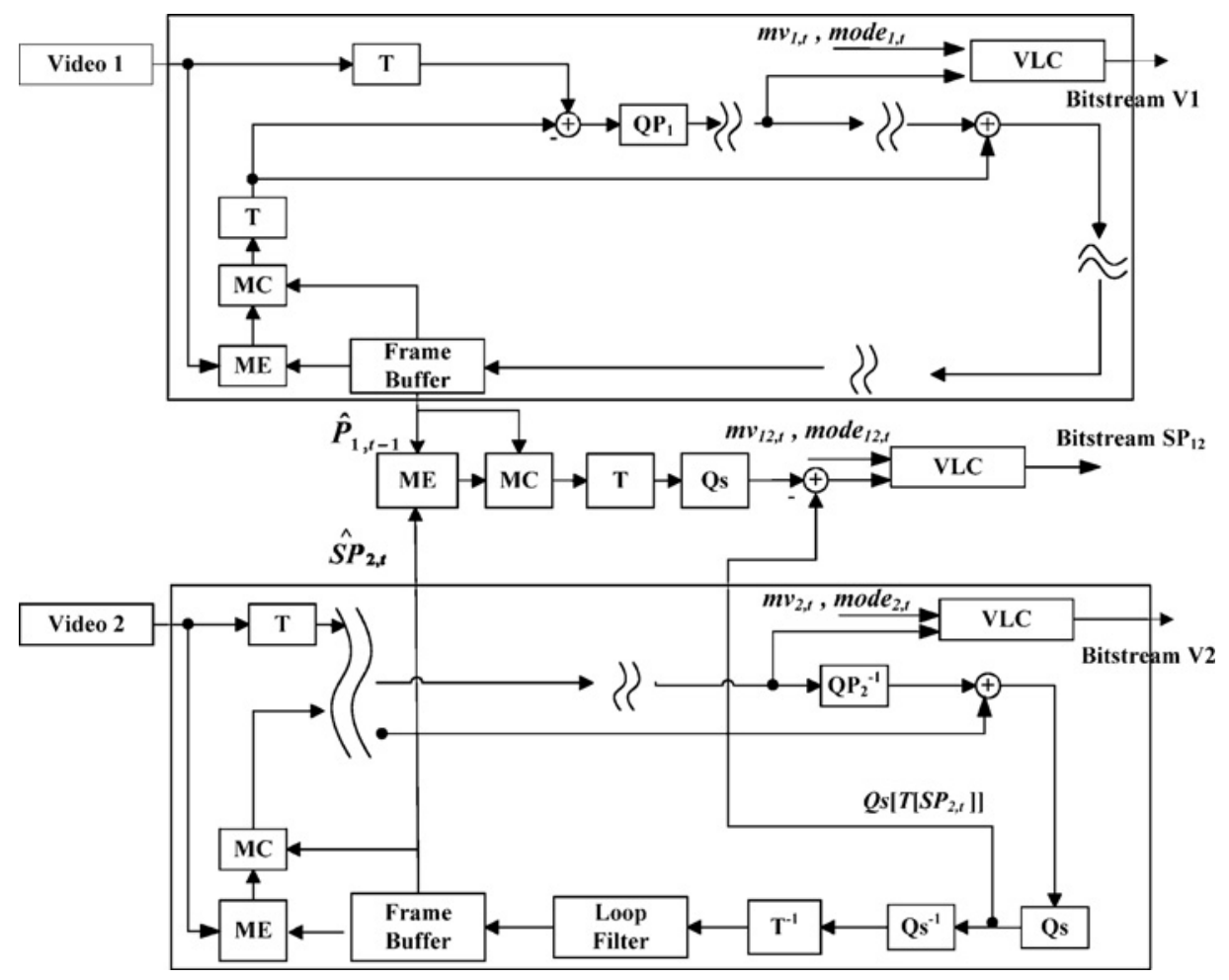

Fig. 3. Motion estimation and compensation of a secondary SP-frame encoder [8].

and its general equation is given by

$$
\begin{aligned}
& J_{\text {mode }}\left(s, c, \operatorname{mode}_{12}, \lambda_{\text {mode }}\right) \\
& =\operatorname{SSD}\left(s, c, \operatorname{mode}_{12}\right)+\lambda_{\text {mode }} \cdot R_{\text {mode }}\left(s, c, \operatorname{mode}_{12}\right)
\end{aligned}
$$

where $\lambda_{\text {mode }}$ is the Lagrangian multiplier for mode decision, mode $_{12}$ is one of the candidate modes during motion estimation, SSD is the sum of the squared differences between $s$ and its reconstruction block $c$, and $R_{\text {mode }}(s, c$, mode 12 ) represents the number of coding bits associated with the chosen mode. To compute $J_{\text {mode }}$, forward and inverse integer transforms, and variable length coding are performed. In the implementation of H.264 codec such as JM11.0 [20], motion estimation of the secondary SP-frame uses $\hat{P}_{1, t-1}$ and the original $S P_{1, t}$ as the reference and target frames, respectively. This arrangement allows the reuse of coding modes (mode ${ }_{1, t}$ in Fig. 1) and motion vectors ( $m v_{1, t}$ in Fig. 1) during secondary SP-frame encoding. It means that

$$
m v_{12, t}=m v_{1, t}
$$

and

$$
\operatorname{mode}_{12, t}=\operatorname{mode}_{1, t} \text {. }
$$

However, the reuse of coding modes and motion vectors reduces the coding efficiency of a secondary SP-frame since the purpose of the secondary SP-frame is to reconstruct $S P_{2, t}$ instead of $S P_{1, t}$. In [8], a secondary SP-frame is encoded to match the exact target frame (reconstructed $S P_{2, t}, \hat{S P}_{2, t}$ ) based on the exact reference $\left(\hat{P}_{1, t-1}\right)$, as depicted in Fig. 3. By using the correct target and reference frames, better compression performance of the secondary SP-frames can be achieved.
Note that the computational complexity evidently increases without reusing coding modes and motion vectors. Nevertheless, secondary SP-frames are always generated in off-line for bitstream switching applications. Thus, complexity is not the major concern for coding secondary SP-frames.

\section{SIZE REDUCTION OF SECONDARY SP-FRAMES IN QDCT-DOMAIN MOTION-COMPENSATED PREDICTION}

By appropriately choosing reference and target frames for mode selection and motion estimation [8], the size of secondary SP-frames can be reduced for the representation of MBV. However, we found that the storage requirement for multi-view video becomes large when secondary SP-frames are acted as the bridging frames for viewpoint switching in MVV streaming. The average storage requirements of secondary SP-frames applied to MBV and MVV applications are shown in Table I where the testing multiview video streams used for the simulation are Ballroom, Exit, Vassar, and Racel with a length of 100 frames. For each MBV, we only selected the first view for the simulation, and the quantization parameters of the high bitrate video $\left(Q_{P 1}\right)$ and the low bitrate video $\left(Q_{P 2}\right)$ were set to 24 and 28 , respectively. On the other hand, for each MVV, the first and second views were used for the simulation. To have a fair comparison, the quantization parameters ( $Q_{P 1}$ and $Q_{P 2}$ in Fig. 3) were also set to 24 and 28, respectively. For both cases, $\hat{P}_{1, t-1}$ and $S P_{2, t}$ are used as the reference frame and the target frame, respectively. From Table I, we can observe that the bits required in coding secondary SP-frames of MVV are much higher than those of MBV. The rise in the bit-counts of MVV is due to the reason that the conventional motion estimation and compensation 


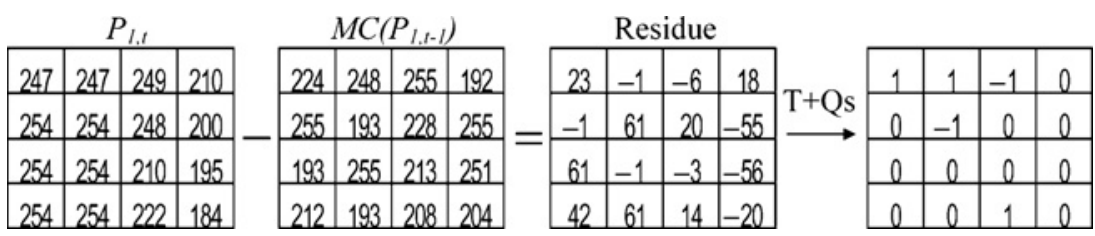

Fig. 4. Motion-compensated prediction using pixel-domain motion estimation in encoding a P-frame.

TABLE I

AVERAGE BIT-COUNTS OF SECONDARY SP-FRAMES IN MVV AND MBV

\begin{tabular}{|l|c|c|}
\hline Sequences & MVV $(\mathrm{kb})$ & MBV $(\mathrm{kb})$ \\
\hline Ballroom & 353 & 221 \\
\hline Exit & 176 & 124 \\
\hline Vassar & 275 & 201 \\
\hline Race1 & 258 & 214 \\
\hline
\end{tabular}

processes work ineffectively in secondary SP-frame coding. It is because the inter-viewpoint disparity in MVV causes an increase in the residue between $\hat{P}_{1, t-1}$ and $S P_{2, t}$ even though the presence of disparity between views can be reduced by correctly selecting reference and target frames. In other words, for coding the secondary SP-frame $S P_{12, t}$ using [8], as shown in Fig. 3, the reference frame and the target frame are $\hat{P}_{1, t-1}$ and $S P_{2, t}$, respectively, and they come from different viewpoints of the same scene. It implies that both temporal motion and inter-view disparity exist between the reference and target frames in MVV. On the other hand, for MBV, both reference and target frames represent the same viewpoint of the scene and the only discrepancy is the quantization parameter used in the formation of the encoded bitstreams. Therefore, the correlation between the reference and target frames of MBV is always larger than that of MVV in secondary SP-frame coding.

In the following, we explain the deficiency in using the conventional motion estimation and compensation processes, which are operated in the pixel domain, for secondary SPframes. In the H.264 codec [20], the motion estimation process in P-frames and secondary SP-frames are the same. Fig. 4 illustrates the step of encoding a block in a P-frame using pixeldomain motion estimation. In this case, quantized coefficients of the P-frame are computed as $Q s\left[T\left[P_{1, t}-M C\left(\hat{P}_{1, t-1}\right)\right]\right]$, where $\mathrm{MC}()$ is the motion-compensation operator. Since the transformation and quantization processes are performed on the residue, $P_{1, t}-M C\left(\hat{P}_{1, t-1}\right)$, most of the coefficients become zero after transformation and quantization. This property paves the way for entropy coding. However, in Fig. 3, the encoding of a secondary SP-frame involves first the transformation and quantization processes of the original $S P_{2, t}$ and $\hat{P}_{1, t-1}$. Then, the quantized coefficients of the secondary SP-frame at $t$, $Q s\left[T\left(S P_{12, t}\right)\right]$, can be obtained as

$$
Q s\left[T\left[S P_{12, t}\right]\right]=Q s\left[T\left[S P_{2, t}\right]\right]-Q s\left[T\left[M C\left(\hat{P}_{1, t-1}\right)\right]\right] .
$$

Fig. 5 uses the same example in Fig. 4 again to show the residue of a secondary SP-frame in which a block is transformed and quantized prior to calculating the residue. In this case, their quantized-transform coefficients are only near, but not equal, resulting in generating many non-zero residues,

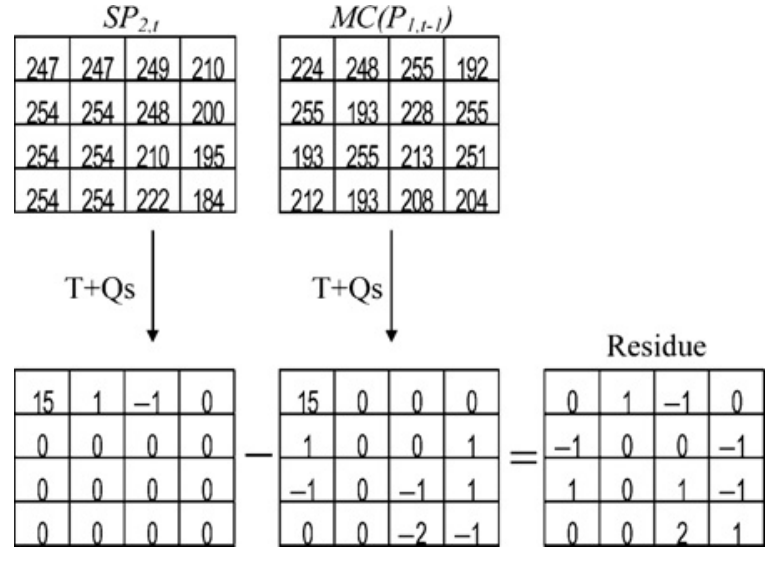

Fig. 5. Motion-compensated prediction using pixel-domain estimation in encoding a secondary SP-frame.

especially for a small $Q s$. Since there is no further quantization from this point, these coefficients should be encoded completely. In entropy coding, the spread of non-zero coefficients exists, a significant amount of bits is required. Therefore, the size of secondary SP-frames becomes large, and this explains the situation in which the pixel-domain motion estimation is not sufficient enough for coding secondary SP-frames. This case always happens in multiview video sequences since V1 and V2 are from different views and their correlation becomes lower, despite the fact that the motion estimation scheme using the correct reference and target frames in V1 and V2 can exploit both motion and disparity in MVV. Figs. 6(a) and 7(a) show the energy distribution of quantized-transform coefficients for the Ballroom sequence in the MBV and MVV applications, respectively, when the scheme in [8] is adopted. Notice that the DC coefficients for these plots are omitted since the value of DC coefficients is relatively large and it affects the plotting scale. Again, for both cases, $Q_{P 1}$ and $Q_{P 2}$ were set to 24 and 28 , respectively, in order to ensure the difference only comes from the presence of disparity between views. From these figures, it is clear to show that there are substantial values of quantized-transform coefficients in MVV as compared with those in MBV, which reduces the compression ratio of secondary SP-frames. It is confirmed that a smart scheme for secondary SP-frame coding is necessary to reduce these quantized-transform coefficients in bitstream switching, especially in multiview video systems. In this paper, we thus propose performing motion estimation and compensation in the QDCT-domain rather than the pixel domain to improve the coding efficiency of secondary SP-frames.

From (5), to optimize the coding of $Q s\left[T\left[S P_{12, t}\right]\right]$ for better entropy coding, quantized-transform domain $\left(Q s\left[T\left[S P_{2, t}\right]\right]-\right.$ 


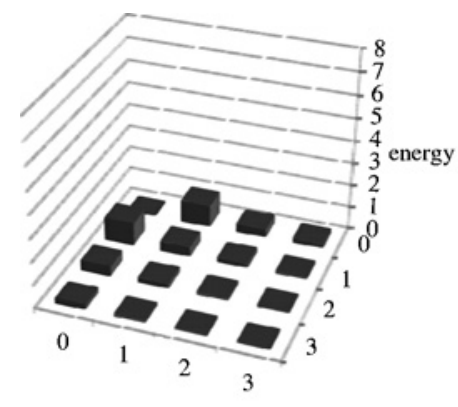

(a)

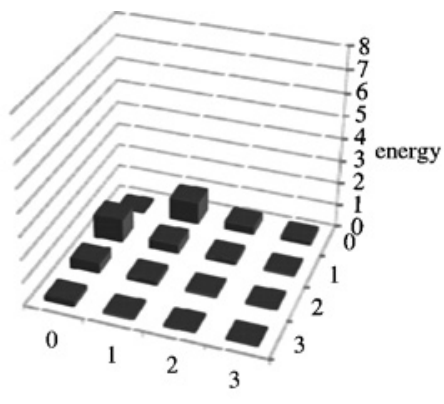

(b)

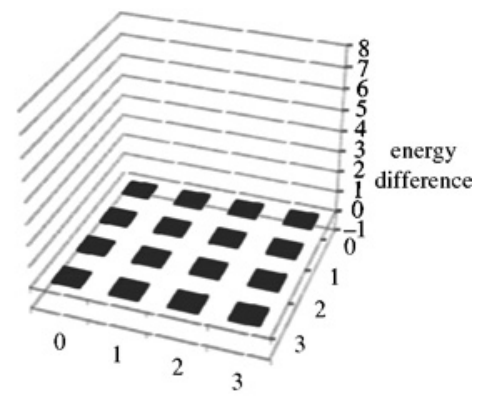

(c)

Fig. 6. Energy distribution of quantized-transform coefficients in the residue for the Ballroom sequence in MBV. (a) Scheme in [8]. (b) Proposed scheme. (c) Their difference.

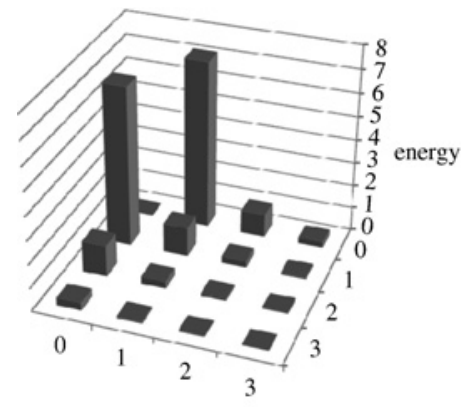

(a)

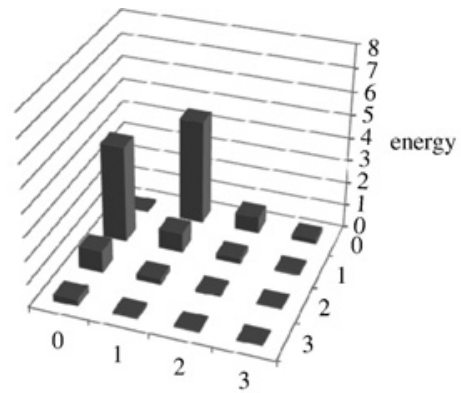

(b)

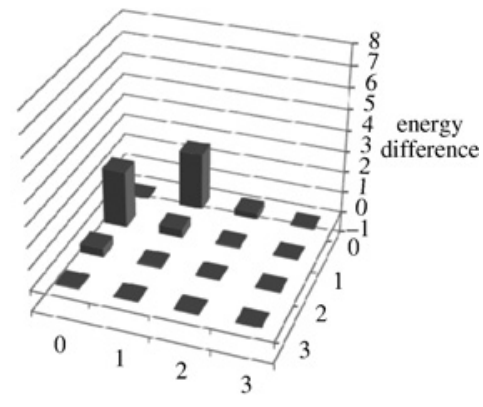

(c)

Fig. 7. Energy distribution of quantized-transform coefficients in the residue for the Ballroom sequence in MVV. (a) Scheme in [8]. (b) Proposed scheme. (c) Their difference.

$\left.Q s\left[T\left[M C\left(\hat{P}_{1, t-1}\right)\right]\right]\right)$ instead of its pixel-domain counterpart should be minimized. However, the Lagrangian cost function $J_{\text {motion }}$ in (1) computes SAD, which is the sum of absolute differences between pixels of the original block $s$ and its reference block $r$. Fig. 5 reveals that this pixel-domain distortion measure is not appropriate for coding secondary SP-frames. Consequently, a quantized-transform domain motion estimation (QDCT-ME) technique that minimizes $Q s\left[T\left[S P_{2, t}\right]\right]-$ $Q s\left[T\left[M C\left(\hat{P}_{1, t-1}\right)\right]\right]$ is adopted in coding secondary SP-frames. In the proposed QDCT-ME, the new Lagrangian cost function $J_{\text {motion }}^{\prime}$ is based on the sum of absolute differences in quantized-transform coefficients and the estimated rate. This new cost function is expressed as

$$
\begin{aligned}
& J_{\text {motion }}^{\prime}\left(m v_{12}, \lambda_{\text {motion }}\right) \\
& =k \cdot \operatorname{SAQTD}(s, r)+\lambda_{\text {motion }} \cdot R_{\text {motion }}\left(m v_{12}-p m v_{12}\right)
\end{aligned}
$$

where $\operatorname{SAQTD}(s, r)$ denotes the sum of absolute differences between the quantized-transform coefficients of the original block $s$ and the quantized-transform coefficients of its reference block $r$, and it can be defined as

$$
S A Q T D(s, r)=\sum|Q s[T(s)]-Q s[T(r)]|
$$

and $R_{\text {motion }}\left(m v_{12}-p v_{12}\right)$ is the estimated number of bits for motion vectors by using a simple table-lookup method. From (6) and (7), $\operatorname{SAQTD}(s, r)$ requires a real quantization process to calculate the sum of absolute differences. But, the Lagrangian multiplier, $\lambda_{\text {motion }}$, is specifically designed for SAD, which is not affected by quantization. It is important to note that the proposed $\operatorname{SAQTD}(s, r)$ is operated in the quantized discrete cosine transform (DCT) domain and the extra quantization causes the energy is no longer preserved although the integer transform itself is an orthonormal transform. After absorbing $Q s$ into the new cost function of the proposed QDCT-ME, $k$ in (6) is a weighting factor to compensate for the energy loss of $\operatorname{SAQTD}(s, r)$ due to the quantization, which can be predefined by empirical results. It is noted that a new $\lambda_{\text {motion }}$ can be set and combined with $k$.

Similar to pixel-domain motion estimation, QDCT-ME supports variable block sizes (from $4 \times 4$ to $16 \times 16$ ). Fig. 8 (a) illustrates how to perform QDCT-ME for a $4 \times 4$ block in which a macroblock is subdivided into sixteen $4 \times 4$ blocks. Each current $4 \times 4$ block is transformed and quantized to $Q s[T(s)]$, of course, in terms of $4 \times 4$ transform. A search window in the reference frame centered on the current $4 \times 4$ block position is searched. QDCT-ME starts with transforming and quantizing a $4 \times 4$ block, $Q s[T(r)]$, in the top right-hand corner of the search window. After obtaining $Q s[T(s)]$ and $Q s[T(r)]$, $S A Q T D(s, r)$ and $J_{\text {motion }}^{\prime}\left(m v_{12}, \lambda_{\text {motion }}\right)$ in (6) can be calculated for this candidate. Then, the next candidate is another $4 \times 4$ block shifted by one pixel in horizontal direction, as depicted in Fig. 8(a), and this block is also $4 \times 4$ transformed and quantized for computing its $J_{\text {motion }}^{\prime}\left(m v_{12}, \lambda_{\text {motion }}\right)$. These procedures continue for all possible candidates within the search window. Note that all possible candidates also need to be transformed and quantized in the QDCT domain. As a consequence, the candidate that minimizes $J_{\text {motion }}^{\prime}\left(m v_{12}, \lambda_{\text {motion }}\right)$ is chosen as the best match. For an $8 \times 8$ block, it contains four $4 \times 4$ 

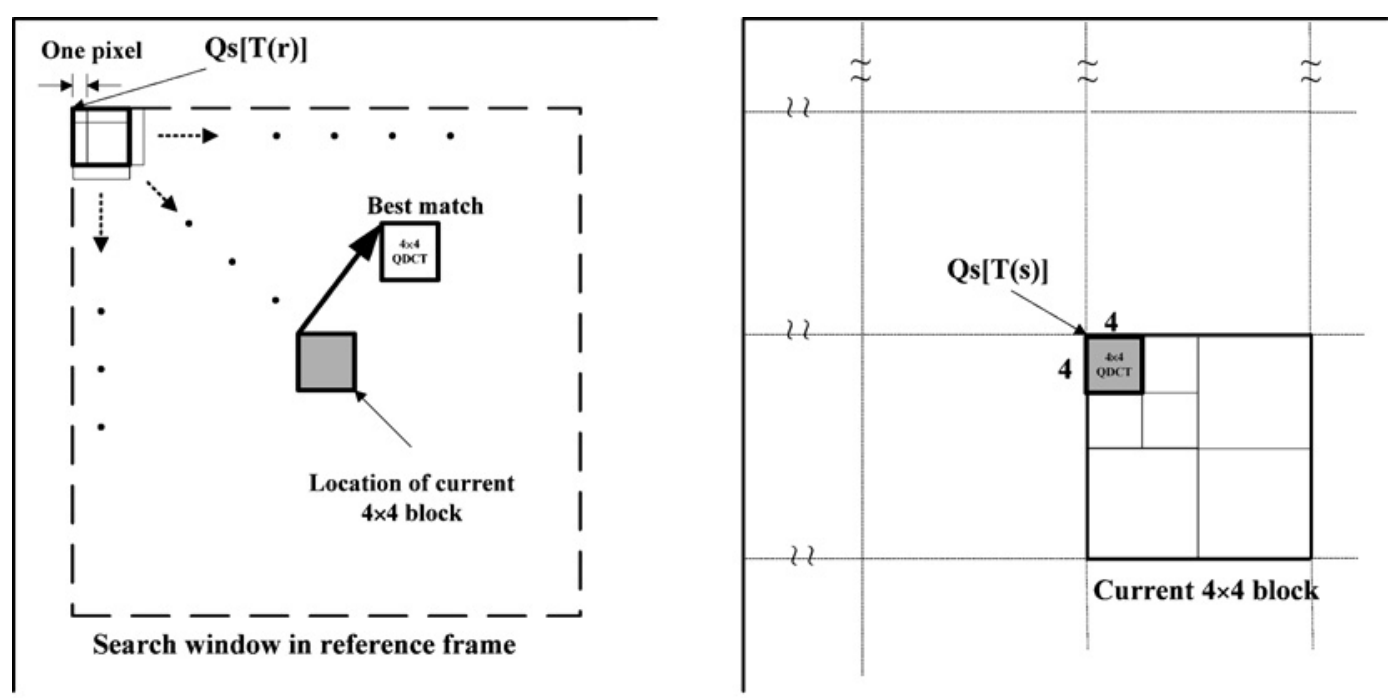

(a)
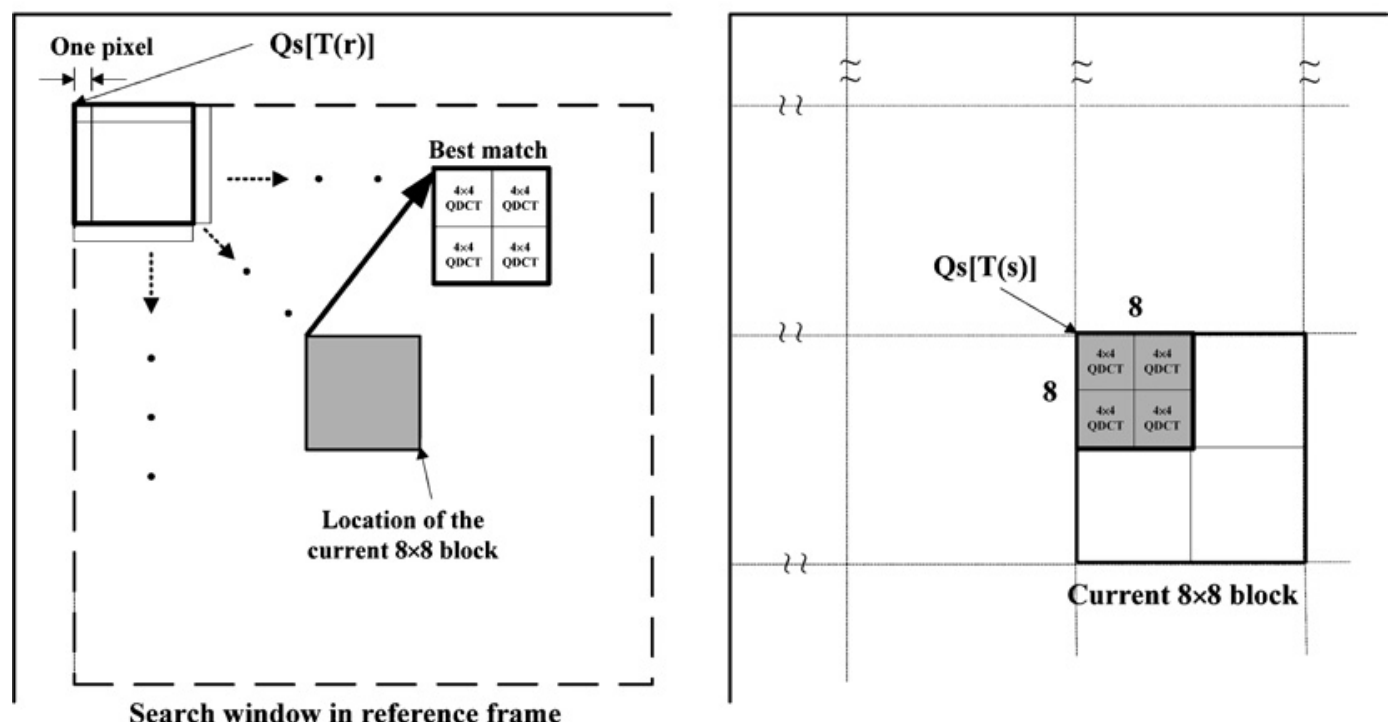

(b)

Fig. 8. QDCT-domain motion estimation on (a) $4 \times 4$ sub-block and (b) $8 \times 8$ sub-block.

blocks, and all these $4 \times 4$ blocks are $4 \times 4$ transformed and quantized to $Q s[T(s)]$, as depicted in Fig. 8(b). These four $4 \times 4$ quantized-transform coefficients are used as a whole to compute $S A Q T D(s, r)$. For other block size, a similar $4 \times 4$ transform is also applied.

For coding a secondary SP-frame, the proposed cost function can find a motion vector and mode for minimizing the residue, $Q s\left[T\left[S P_{12, t}\right]\right]$, in (5). Note that $S A Q T D$ is computationally intensive since all the pixel blocks are necessary to be transformed and quantized to the quantized-transform domain. Its computational complexity is now examined. One straightforward approach to implement the proposed cost function in (6) is to directly perform the transformation and quantization, on the fly, during $S A Q T D$ calculation. That is, for each block, the additional transformation and quantization on $Q s[T(s)]$ and all possible candidates of $Q s[T(r)]$ within the search window are required. This straightforward implementation results in repetition of the same calculation of $Q s[T(r)]$ because the search windows of neighboring blocks are overlapped. This repetition increases linearly with the size of search window. To avoid this repetitive transformation and quantization, all possible locations of $Q s[T(r)]$ can precalculated and stored in the buffer. By exploiting the reusage of $Q s[T(r)]$, the computation of $S A Q T D$ can be reduced drastically. From Fig. 8 , only $4 \times 4$ block size is considered in our implementation. It means that $4 \times 4$ blocks are transformed to the quantized-transform domain. Let $\mathrm{QT}_{4 \times 4}$ be a notation of total number of operations involved in transforming and quantizing a $4 \times 4$ reference block, which is taken as a unit to measure and compare the extra computational complexity of the proposed $S A Q T D$. The size of the video frame is supposed to be $\mathrm{W} \times \mathrm{H}$. To pre-calculate all possible locations of $Q s[T(r)]$ in the buffer, the proposed $S A Q T D$ requires additional $(\mathrm{W}-3) \times(\mathrm{H}-3) \mathrm{QT}_{4 \times 4}$ operations. It is also noted that 


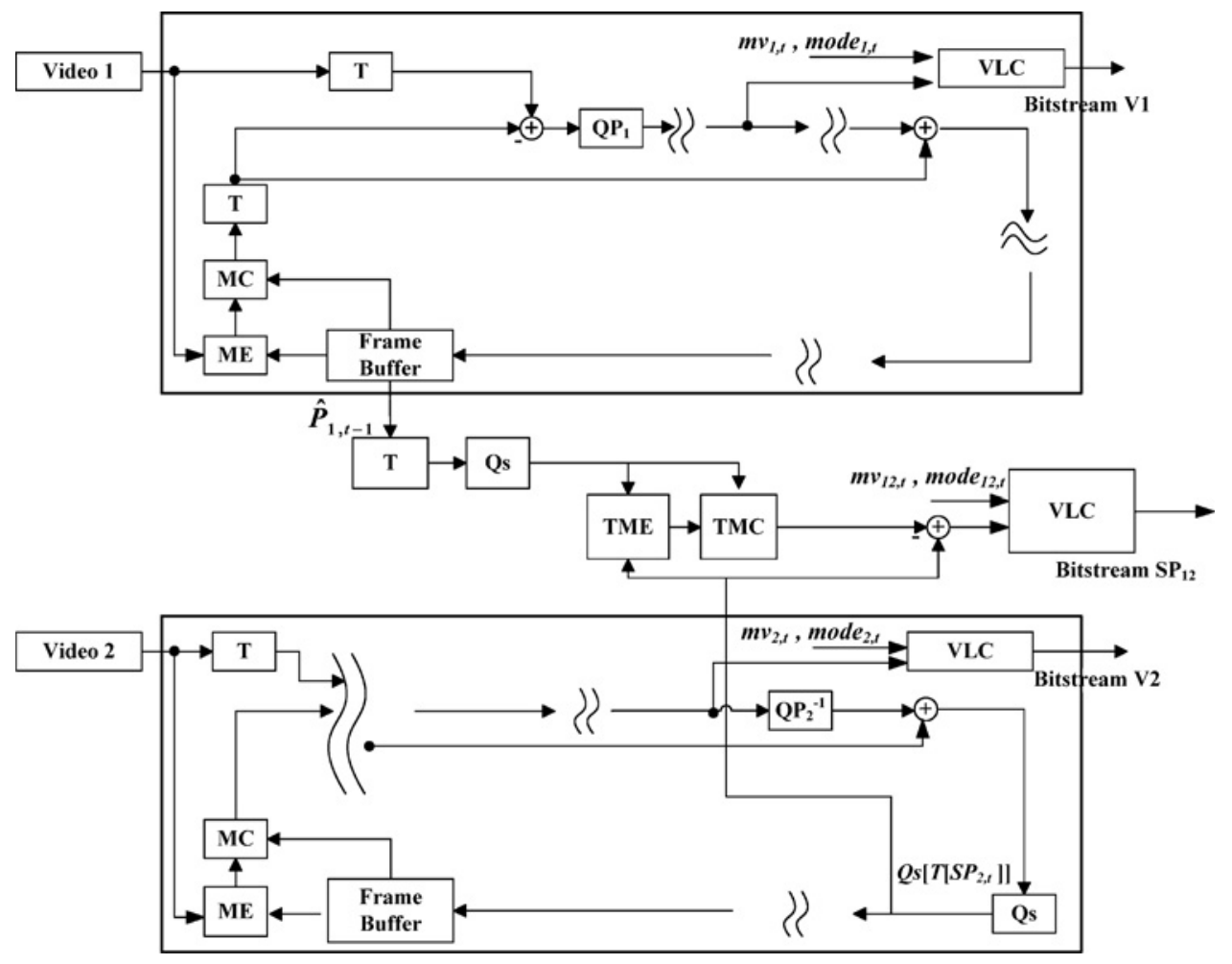

Fig. 9. Proposed secondary SP-frame encoder in the QDCT domain.

the size of the additional buffer is $4(\mathrm{~W}-3) \times 4(\mathrm{H}-3)$. For other block types in motion estimation, this additional buffer can also be re-used. Besides, some phase correlation techniques operated in the DCT domain were designed in [21], [22] to estimate motion vectors in the MPEG standard. This phase correlation technique is also possible to expedite our proposed QDCT-ME. Further investigation is necessary since the QDCT$\mathrm{ME}$ is operated in the QDCT domain while the technique in [21], [22] is developed in the DCT-domain only. However, the complexity is not the major concern for secondary SPframe coding since this frame type is always encoded offline for bitstream switching applications. On the other hand, the accuracy of the cost function enhances the coding efficiency of secondary SP-frames which leads to a significant reduction of the storage requirement in the video server.

In summary, the pixel-domain motion estimation is to minimize the absolute differences in which the subtraction is performed in terms of pixels while the quantized-transform domain motion estimation is to minimize the absolute differences in which the subtraction is performed in terms of transformed and quantized coefficients.

\section{Simulation Results}

For the implementation, Fig. 9 shows the block diagram of applying our new quantized-transform domain motion estimation technique in the secondary SP-frame encoder. The reference and target frames in the quantized-transform domain are the inputs of QDCT-ME. After the motion vectors for each block are obtained through (6), a corresponding quantized-transform domain motion compensation
(QDCT-MC) is used to compute the $Q s\left[T\left[M C\left(\hat{P}_{1, t-1}\right)\right]\right]$. With $Q s\left[T\left[M C\left(\hat{P}_{1, t-1}\right)\right]\right]$ and $Q s\left[T\left[S P_{2, t}\right]\right]$, as depicted in Fig. 9, the residue $Q s\left[T\left[S P_{2, t}\right]\right]$ can then be calculated.

To evaluate the performance of the proposed scheme, this section presents the simulation results in both MVV and MBV. For MVV, five test sequences with eight views, Ballroom $(640 \times 480$, from MERL [23]), Exit $(640 \times 480$, from MERL [23]), Vassar $(640 \times 480$, from MERL [23]), Racel $(640 \times 480$, from KDDI [24]), and Breakdancers $(1024 \times 768$, from Microsoft) were used for performance comparison in coding secondary SP-frames. Ballroom, Exit, and Vassar were captured by 1D parallel-aligned cameras while the camera arrangement of Breakdancers was 1D arc. In addition, Racel was produced by the moving set but fixed relative positions of cameras by KDDI [24]. Notice that the eight views in Breakdancers and Racel were non-rectified whereas those in other sequences were rectified. For MBV, two single-view sequences with complex motion, Riverbed $(704 \times 480)$ and Crew $(1280 \times 720)$, were used to demonstrate the performance of the proposed scheme. In each sequence, 100 frames were encoded by the H.264 reference codec (JM version 11.0) [20]. In our experiments, extended profile with context-adaptive variable-length coding (CAVLC) entropy encoding was used to configure the encoder. RD optimization was enabled. For the motion estimation process, a search range of 32 was set for both P-frames and SP-frames. For the proposed scheme, the scaling factor $k$ of (6) was set to 3, which was found by experimental observations. There were two sets of experiments carried out. First, we focus on the performance of the proposed QDCT-domain motion-compensated prediction technique applying to secondary SP-frame coding in multiview 


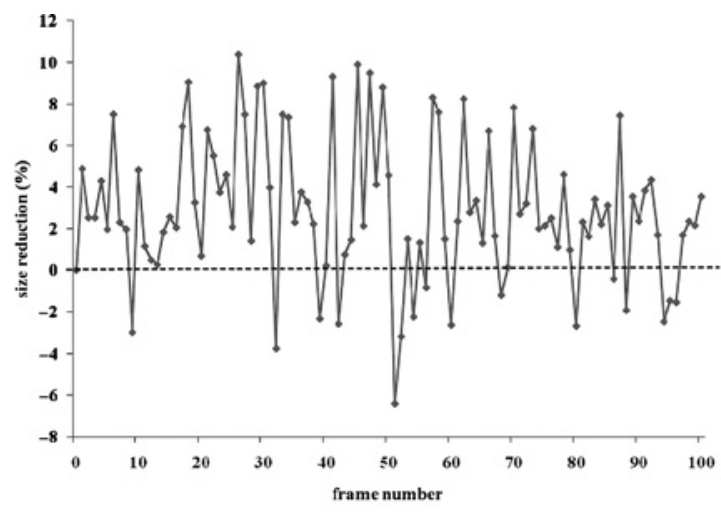

(a)

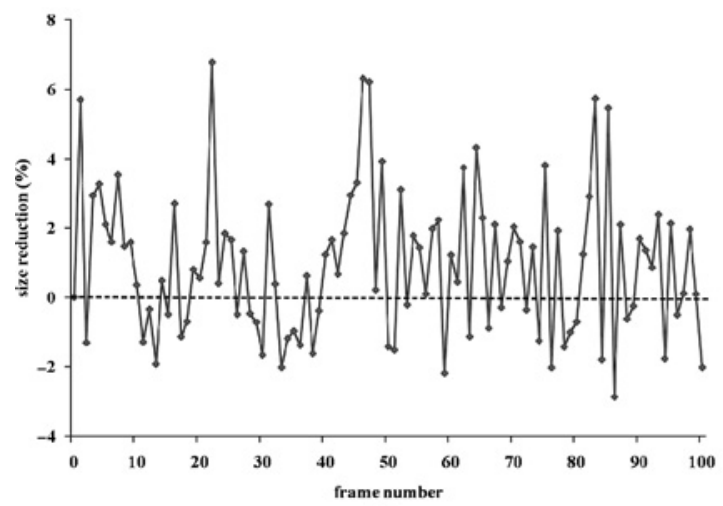

(c)

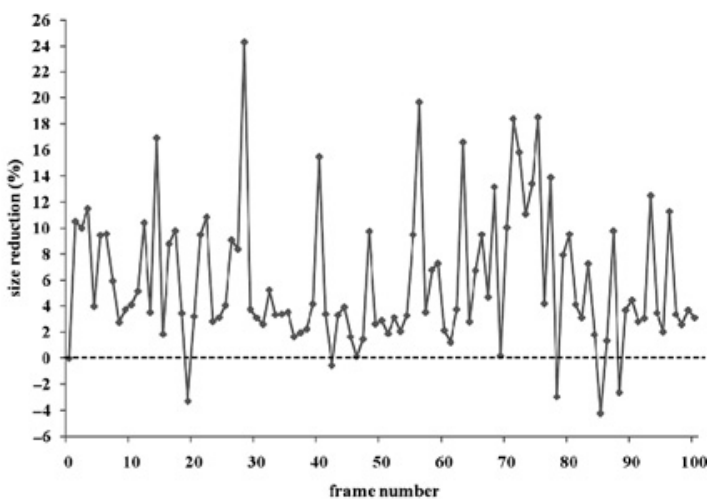

(b)

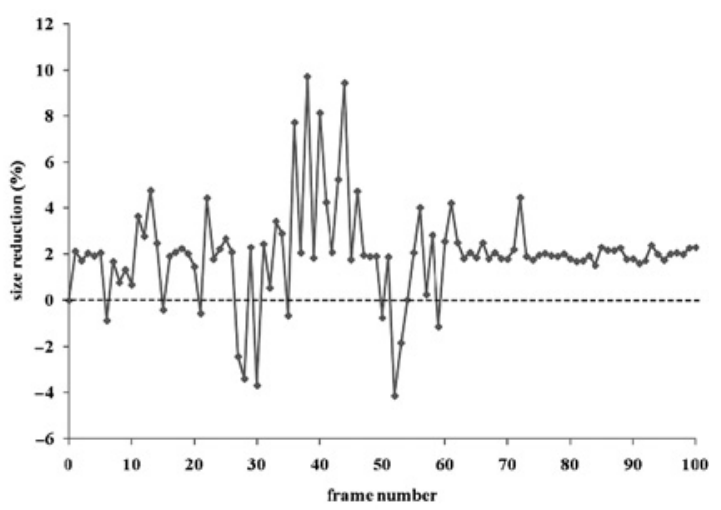

(d)

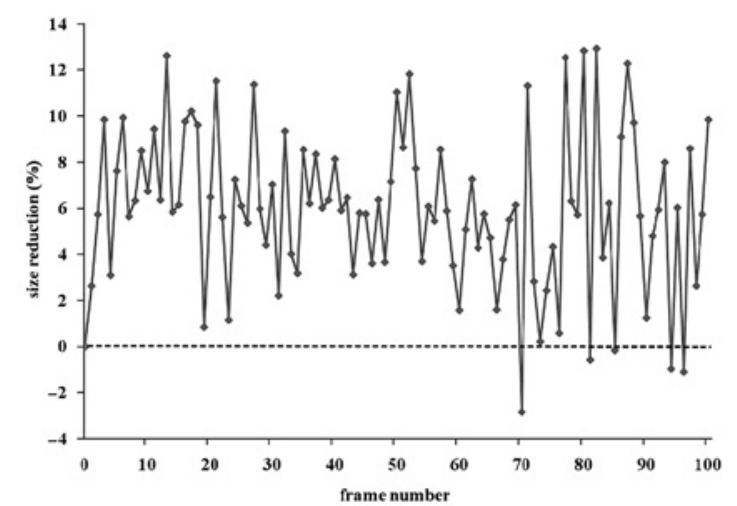

(e)

Fig. 10. Frame-by-frame comparisons of size reduction of secondary SP-frames in percentage achieved by the proposed scheme over the scheme in [8] at $Q_{P}=20$. (a) Ballroom. (b) Exit. (c) Vassar. (d) Race1. (e) Breakdancers.

videos and single-view videos. Second, we demonstrate the storage reduction in a multiview video coding system with the proposed technique. In both experiments, results are compared to the scheme in [8]. To make the comparison impartial, both schemes employed a full search algorithm for motion estimation.

\section{A. Performance of the Proposed QDCT-Domain Motion- Compensated Prediction Technique in Secondary SP-Frame Coding}

1) Viewpoint Switching in Multiview Video: The first set of experiments aims at evaluating the coding efficiency of secondary SP-frames based on our proposed QDCT-domain motion-compensated prediction. For simplicity but without loss of generality, only two views (view 1 and view 2) were selected to perform switching using SP-frames. These two different bitstreams from two different viewpoints were encoded with the same sets of $Q_{P 1}, Q_{P 2}$, and $Q_{S}$. $Q_{P 1}$ and $Q_{P 2}$ were equal for encoding these two views, that is, $Q_{P 1}=Q_{P 2}=Q_{P}$. They were then set to 28 whereas $Q_{S}$ was set to $22\left(Q_{P}-6\right)$, which is the optimal setting according to [5]. To have a comprehensive and fair comparison between the proposed scheme and the scheme in [8], we did exhaustive simulation on the bit-counts of all possible secondary SPframes switching from view 1 to view 2 . That is, only the first frames of the two views were encoded as I-frames, and all the rest frames were encoded in turn as SP-frames while non-switching frames were encoded as P-frames. 


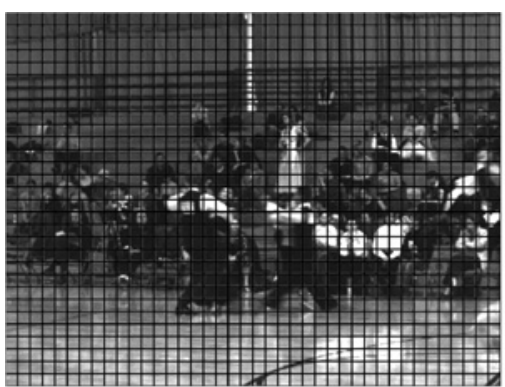

(a)

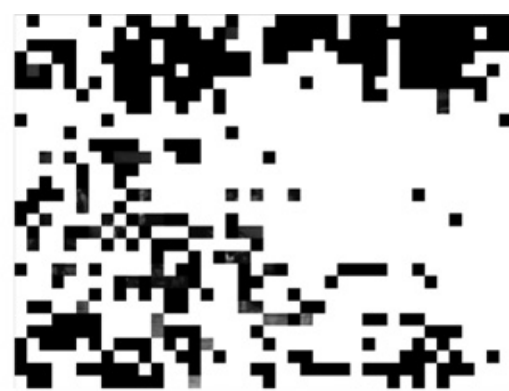

(b)

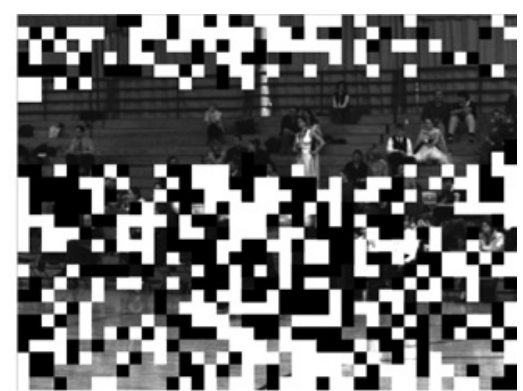

(c)

Fig. 11. (a) Reference frame in view 1 of Ballroom. Bit-count comparison in macroblock level between the proposed scheme and the scheme in [8] in (b) MVV and (c) MBV. Macroblocks filled with white color and black color, which represent our proposed scheme, have superior and inferior performances as compared with the scheme in [8], respectively.

Fig. 10(a)-(e) show a frame-by-frame comparison of size reduction in secondary SP-frames for different multiview sequences. In this figure, the values of the $y$-axis mean the average size reduction of secondary SP-frames in percentage of our proposed scheme over the scheme in [8] whereas the negative values mean the proposed scheme generates more bit-count as compared with [8]. From Fig. 10(a) to (e), the proposed scheme can overwhelmingly reduce the size of secondary SP-frames of up to $11 \%, 25 \%, 7 \%, 10 \%$, and $13 \%$ in the SP-frames of Ballroom, Exit, Vassar, Race1, and Breakdancers, respectively. The significant improvement of the proposed scheme is due to the benefit of performing motion estimation and compensation in the QDCT domain. In [8], even though a proper target frame is selected for motion estimation, the performance is still not good enough. It is due to the reason that only the conventional pixel-domain motion estimation technique is employed for coding the secondary SPframes. In this situation, most of the quantized-transform coefficients become non-zero after transformation and quantization, as shown in Fig. 5, which unfavor the use of entropy coding. Consequently, more bits are required to encode secondary SP-frames. On the other hand, our proposed scheme produces secondary SP-frames using motion estimation in the QDCT domain. The transformed and quantized coefficients are used to calculate the distortion in the Lagrangian cost function. The new SAQTD is able to find the motion vectors with more zero values of quantized-transform coefficients which benefits the entropy coding of secondary SP-frames. This provides a remarkable size reduction of our proposed scheme. Note that, as shown in Fig. 10, some secondary SP-frames of the test sequences generated by using the QDCT-domain motion estimation technique introduce more bits as compared with the pixel-domain technique. It is due to the distribution of quantized-transform coefficients in some $4 \times 4$ blocks where all pixels are similar or with the same value. In these blocks, their quantized-transform coefficients are mainly zeros except the DC coefficient. If this type of QDCT-domain blocks is used to search over a predetermined search area on the transformed and quantized reference frame, all the $S A Q T D$ values are very similar and the motion estimation process becomes very sensitive to noise. In this case, the smallest $S A Q T D$ value may not be presumed to be the best motion vector. Thus, the bit-counts of some macroblocks in the secondary SP-frames encoding by pixel-domain motion estimation are possible to be slightly smaller than those encoding by QDCT-domain motion estimation. The evidence is shown in Fig. 11 where the bit-count comparison in all macroblocks of one switching frame between the proposed scheme and the scheme in [8] in Ballroom is illustrated. In this example, the reference frame from view 1 is depicted in Fig. 11(a). In Fig. 11(b), the macroblocks filled with white color are those macroblocks that the proposed scheme requires less bit-count as compared with the scheme in [8], and they are dominated in this frame. On the other hand, the macroblocks filled with black color show the region has inferior performance for our proposed scheme. They are mainly in the regions of the curtain and the floor that contain similar pixel values within the macroblock. However, it only happens in a few macroblocks in which the difference in bit-counts is very tiny. To avoid this, one straightforward way is to perform both pixel-domain and QDCT-domain motion estimation, and select the motion vector with the minimum bit-counts. Notice that the macroblocks without filling any color in Fig. 11(b) represent same bitcounts generated by both schemes.

Fig. 12(a)-(e) also show the average percentage reduction in size of secondary SP-frames with different $Q_{P}$ for various sequences. In this experiment, $Q_{P}$ was varied from 16 to 28 with a stepsize of 4 . These quantization parameters lead to a bitrate variation of the sequences in different viewpoints from approximately $1.4 \mathrm{Mb} / \mathrm{s}$ to $160 \mathrm{~kb} / \mathrm{s}$. Again, $Q_{S}$ was set to $Q_{P}-6$. It is obvious from Fig. 12(a) to (e) that by using the proposed QDCT-domain motion estimation, the size of secondary SP-frames can be remarkably reduced. This large reduction highlights the importance of using a proper domain for motion estimation in secondary SP-frame encoding. Fig. 12(e) also shows that the overall gain of the Breakdancers sequence is higher than the other sequences. The reason is that, in Breakdancers, it is difficult to perform good disparity compensation using translational approximation (i.e. based on block-matching) due to the large baseline distance of camera arrangement. It results in low interview correlation. In this situation, directly minimizing the quantized-transform coefficients by the new SAQTD is more beneficial to the entropy coding of secondary SP-frames.

2) Bitstream Switching in Multiple Bitrate Video: To demonstrate the impact of the proposed scheme on bitstream 


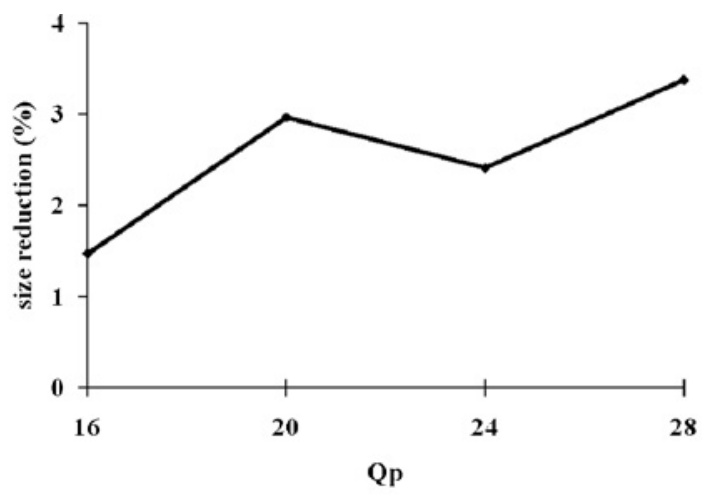

(a)

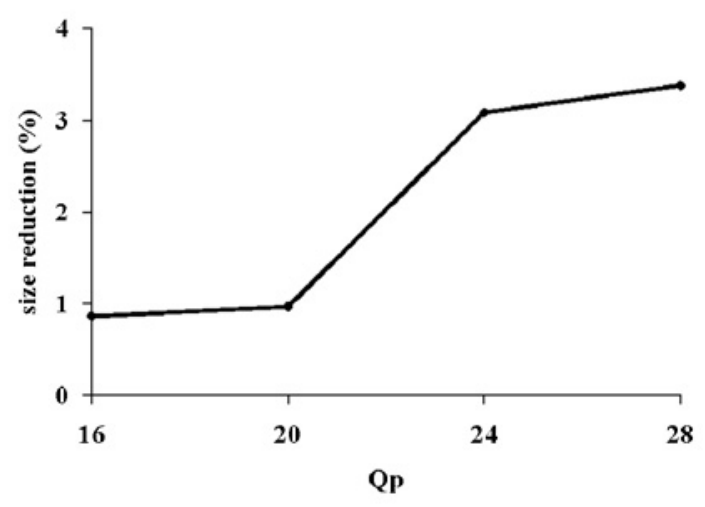

(c)

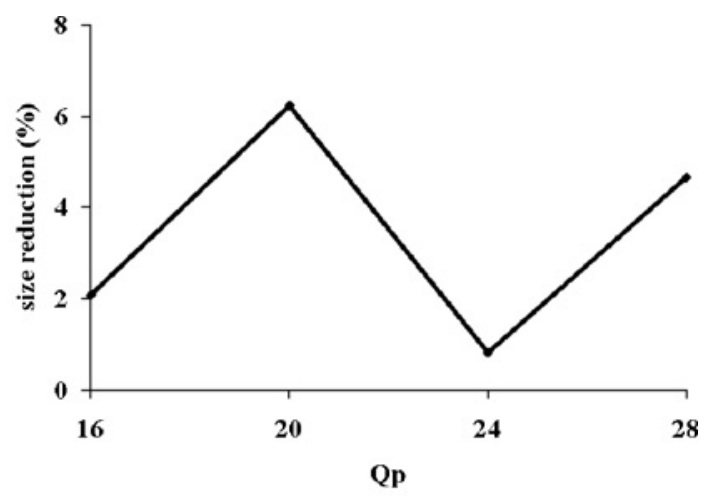

(b)

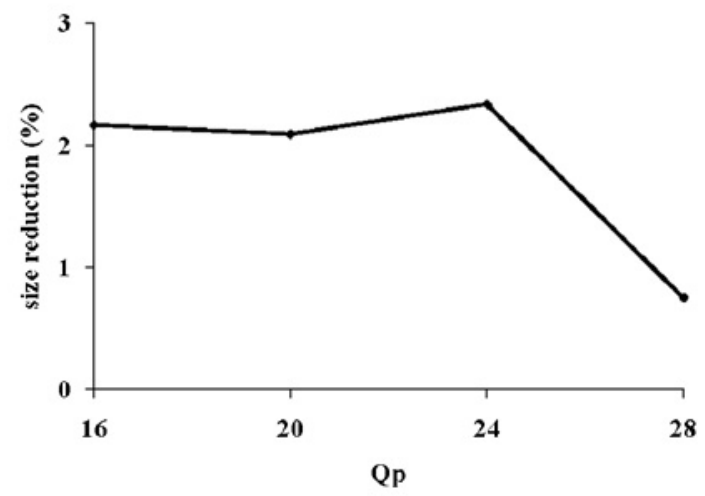

(d)

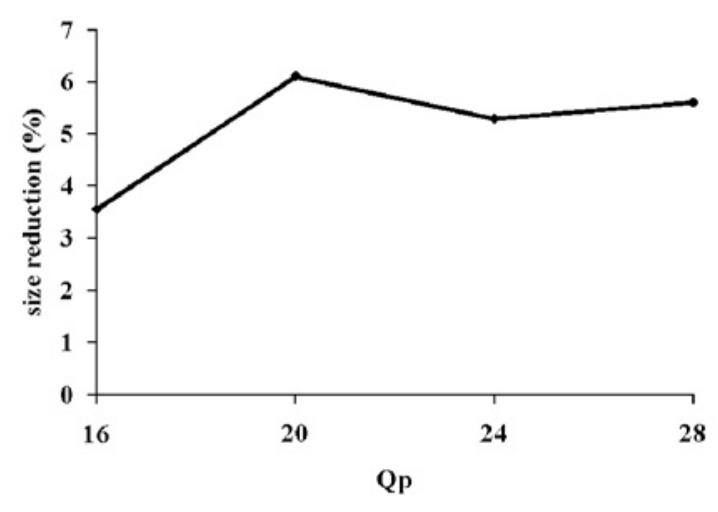

(e)

Fig. 12. Size reduction of secondary SP-frames in percentage achieved by the proposed scheme over the scheme in [8] with different $Q_{P}$. (a) Ballroom. (b) Exit. (c) Vassar. (d) Race1. (e) Breakdancers.

switching in MBV, Fig. 13 gives the comparison of size reduction (in percentage) in secondary SP-frames for Ballroom in MBV and MVV applications. The high and low bitrate bitstreams of MBV were generated by encoding only the first view of Ballroom with $Q_{P 1}$ and $Q_{P 2}$ being equal to 24 and 28, respectively. For MVV, the first and second views were also encoded by setting $Q_{P 1}$ and $Q_{P 2}$ to 24 and 28, respectively, in order to have a fair comparison, as discussed in Section III. As usual, $Q_{S}$ was set to 22 . In Fig. 13, only a very small size reduction of secondary SP-frames is obtained in MBV while the sizes of secondary SP-frames are considerably reduced in MVV. Notice that, in MBV, the bitstream switching takes place from high to low bit rate video. As discussed in Section III, for the scheme in [8], the energy in quantized-transform coefficients is already very small, as depicted in Fig. 6(a). The room for improvement is very limited. Fig. 6(b) also shows the energy distribution of quantized-transform coefficients for using our proposed QDCT-ME. It can be seen that the improvement is not noticeable, as illustrated in Fig. 6(c) in which the distribution of the energy difference between the scheme in [8] and the proposed scheme is computed. The positive values represent that our proposed scheme produces smaller values or magnitudes of coefficients to be encoded. To have a closer look at the macroblock level in the case of MBV, the bitcount comparison of all macroblocks between the proposed scheme and the scheme in [8] is depicted in Fig. 11(c). In the case of MBV shown in Fig. 11(c), although the number of macroblocks filled with white color is more than that of 


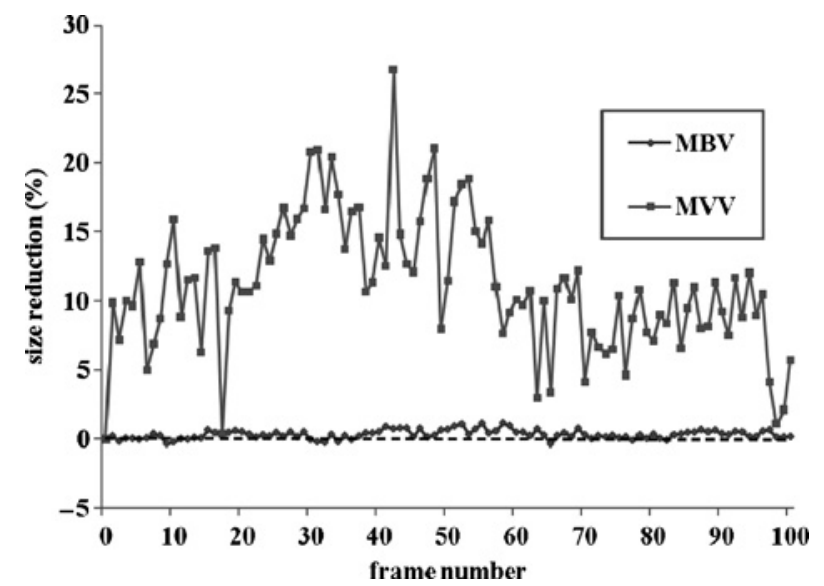

Fig. 13. Size reduction of secondary SP-frames in percentage achieved by the proposed scheme over the scheme in [8] for Ballroom in MBV and MVV.

TABLE II

Percentages of Average Size Reduction of Secondary SP-Frames OF OUR PROPOSED SCHEME OVER THE SCHEME IN [8] IN MBV WITH VARIOUS SKIPPING FACTORS

\begin{tabular}{|c|c|}
\hline No. of Skip Frames & Size Reduction (\%) \\
\hline 0 & 0.4831 \\
\hline 1 & 0.5978 \\
\hline 2 & 0.8374 \\
\hline 3 & 0.9183 \\
\hline
\end{tabular}

the macroblocks filled with black color, it is not remarkable as compared with Fig. 11(b), since many macroblocks in the background region containing audiences produce same bitcounts. It is contrast to the case of MVV where the energy in the quantized-transform coefficients becomes larger, as shown in Fig. 7(a), due to the presence of disparity. This situation favors our proposed $S A Q T D$, which is intended to minimize the residue in the QDCT domain. As a result, a considerable decrease in the energy of quantized-transform coefficients can be achieved, as illustrated in Fig. 7(b) and (c).

It is interesting to note that the proposed SAQTD can perform better in the case of MBV containing scenes with high motion activity. To verify this, for the sake of simplicity, we could simulate high motion scenes in Ballroom by regularly dropping some frames. Table II shows the size reduction in secondary SP-frames for Ballroom in MBV with various skipping factors. It can be seen from this table that the percentages of average size reduction of the secondary SP-frames of our proposed scheme over the scheme in [8] are augmented as more frames are skipped in MBV since the interframe correlation decreases as skipping factor rises.

It is clear that the performance of the proposed SAQTD is determined by the degree of interframe correlation in MBV. The weaker the interframe correlation is, the better the SAQTD can provide. To further demonstrate this, Fig. 14 shows a frame-by-frame comparison of size reduction in secondary SP-frames for the Riverbed and Crew sequences. In this figure, we used the same setting as the case of encoding the single-view video of Ballroom mentioned above. It is observed that the size reduction of secondary SP-frames is

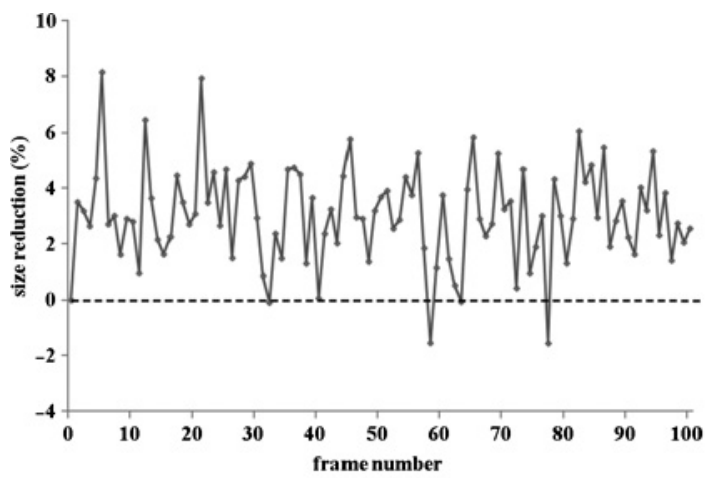

(a)

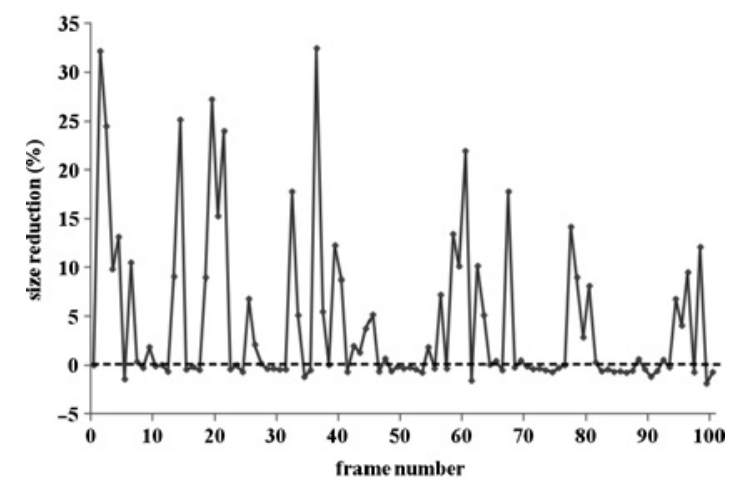

(b)

Fig. 14. Frame-by-frame comparisons of size reduction of secondary SPframes in percentage achieved by the proposed scheme over the scheme in [8] in (a) Riverbed and (b) Crew.

up to $8 \%$ and $33 \%$ in the SP-frames of Riverbed and Crew, respectively. As comparing with the MBV result of Ballroom from Fig. 13, the proposed scheme applying on these two sequences can give more significant improvement. The merit of our proposed scheme is due to the inherent property of these video sequences. In the first view of Ballroom, owing to the frames of MBV being captured by the same camera at the same time in the same scene, the translational motion model could be good enough for practical purposes. Consequently, it generates smaller quantized-transform coefficients in secondary SP-frames. In contrast, Riverbed is the shot of a Riverbed seen through the water while Crew contains NASA crew leaving a building with flashlights. In these sequences, they both include scenes with challenging motion in which translational motion is not able to find the true motion in these scenes. The scheme in [8] cannot find suitable motion vectors in pixel domain. The incorrect motion vectors cause an increase in residue of secondary SP-frames. The evidence is shown in Fig. 15 where the average energy of the residue in quantized-transform domain of Ballroom, Riverbed, and Crew is plotted. In this figure, it can be easily seen that the quantized-transform coefficients in the residue of Riverbed and Crew are much larger than those of Ballroom. It implies that directly minimizing the quantized-transform coefficients using the proposed scheme is more crucial even though the obtained motion vectors are not reflecting the real motion. In other words, the QDCT-domain motion estimation aims at minimizing the quantized-transform coefficients and can 


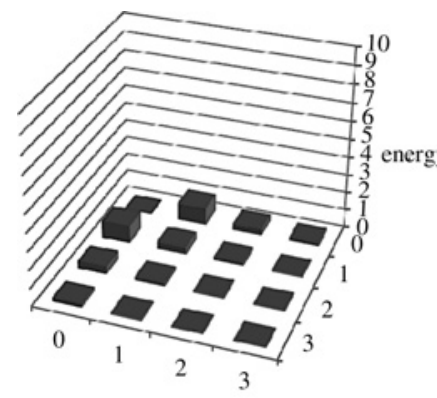

(a)

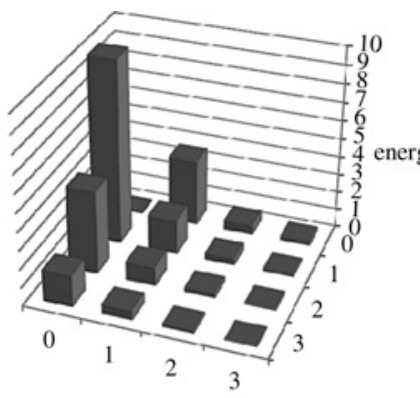

(b)

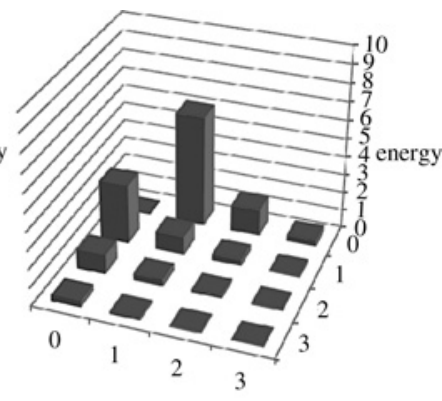

(c)

Fig. 15. Energy distribution of quantized-transform coefficients in the residue for (a) first view of Ballroom, (b) Riverbed, and (c) Crew.

TABLE III

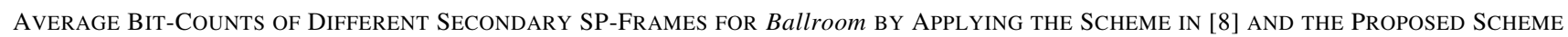

\begin{tabular}{|c|c|c|c|c|c|c|c|c|c|c|c|c|}
\hline$Q_{P}$ & \multicolumn{3}{|c|}{16} & \multicolumn{3}{|c|}{20} & \multicolumn{3}{|c|}{24} & \multicolumn{3}{|c|}{28} \\
\hline & $\begin{array}{l}\text { Scheme } \\
\text { in [8] } \\
(\mathrm{kb})\end{array}$ & $\begin{array}{l}\text { Proposed } \\
\text { Scheme } \\
\text { (kb) }\end{array}$ & $\Delta(\%)$ & $\begin{array}{l}\text { Scheme } \\
\text { in }[8] \\
(\mathrm{kb})\end{array}$ & $\begin{array}{l}\text { Proposed } \\
\text { Scheme } \\
(\mathrm{kb})\end{array}$ & $\Delta(\%)$ & $\begin{array}{l}\text { Scheme } \\
\text { in }[8] \\
(\mathrm{kb})\end{array}$ & $\begin{array}{l}\text { Proposed } \\
\text { Scheme } \\
(\mathrm{kb})\end{array}$ & $\Delta(\%)$ & $\begin{array}{l}\text { Scheme } \\
\text { in }[8] \\
(\mathrm{kb})\end{array}$ & $\begin{array}{l}\text { Proposed } \\
\text { Scheme } \\
(\mathrm{kb})\end{array}$ & $\Delta(\%)$ \\
\hline $\mathbf{0} \rightarrow \mathbf{1}$ & 958 & 947 & 1.1566 & 710 & 695 & 2.0237 & 451 & 448 & 0.7190 & 330 & 304 & 8.0112 \\
\hline $1 \rightarrow 2$ & 897 & 884 & 1.4656 & 648 & 635 & 1.9467 & 405 & 391 & 3.5841 & 282 & 277 & 1.8485 \\
\hline $2 \rightarrow 3$ & 895 & 886 & 1.0328 & 645 & 635 & 1.5428 & 409 & 402 & 1.5861 & 289 & 286 & 1.1210 \\
\hline $3 \rightarrow 4$ & 905 & 896 & 1.0246 & 661 & 651 & 1.4221 & 426 & 416 & 2.2084 & 299 & 295 & 1.3338 \\
\hline $4 \rightarrow 5$ & 882 & 868 & 1.5868 & 633 & 620 & 2.0091 & 410 & 400 & 2.2531 & 293 & 289 & 1.3372 \\
\hline $5 \rightarrow 6$ & 894 & 885 & 1.0013 & 652 & 639 & 2.0189 & 414 & 401 & 3.2270 & 302 & 284 & 5.9924 \\
\hline $6 \rightarrow 7$ & 929 & 919 & 1.1572 & 690 & 677 & 1.9418 & 433 & 426 & 1.6713 & 306 & 302 & 1.1584 \\
\hline $\begin{array}{l}\text { Total } \\
\text { size }\end{array}$ & 6360 & 6283 & 1.2019 & 4639 & 4553 & 1.8455 & 2947 & 2884 & 2.1535 & 2099 & 2035 & 3.0673 \\
\hline
\end{tabular}

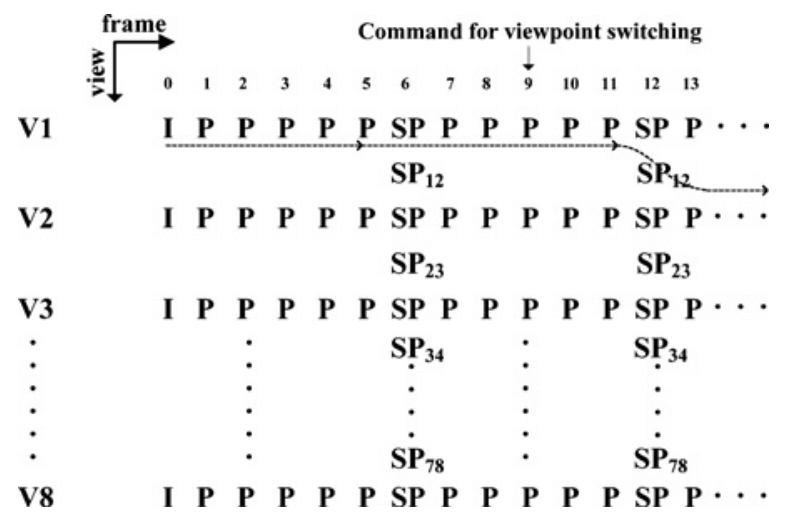

Fig. 16. Multiview video coding system.

provide remarkable improvement over the scheme in [8] when the complex motion exists.

\section{B. Storage Requirement of Secondary SP-Frames in Multiview Video Coding System}

To illustrate more concretely the benefits of adopting the proposed scheme in streaming multiview video, we implemented a multiview video system with the support of lowdelay viewpoint switching. For this practical system, a serverclient architecture was used. The client is connected to the video server over an Internet Protocol (IP) network and requests access to the specific view of the encoded multiview video, which is stored in the server. In the encoded multiview video, SP-frames are inserted periodically at intervals of six frames in each bitstream, as depicted in Fig. 16. In other words, we encoded a primary SP-frame every six frames while other frames were encoded as P-frames. Other settings are the same as those in the above experiments. Besides, all processes of viewpoint switching among eight views are performed at SP-frames only. At each switching point, there are a total number of seven secondary SP-frames that have to be prepared and stored in the server to support viewpoint switching among eight views. This scenario is more applicable for low-delay viewpoint switching in a real multiview video system. Note that only one-way switching direction is assumed in this experiment for simplicity. For viewpoint switching with both directions, another seven secondary SP-frames in the opposite switching direction are added similarly. When a user subscribes to the server, the multiview video service will be provided. Two logical channels are established between the server and the client: the data channel and the control channel. The data channel, implemented by real-time transport protocol (RTP) in our system, is used to deliver the requested bitstream of multiview video. This RTP standardizes the packet format for delivering video over the Internet. In addition, the server receives viewpoint switching command through the control channel, which was implemented by the real time streaming protocol (RTSP). If a viewpoint switching command is received, the server will continue sending video bitstream of the current view direction until reaching the next SP frame. After that, it will send the video bitstream of the new viewpoint from that SP frame. For the example shown in Fig. 16, a user is watching view 1 and can interact with the server. At 


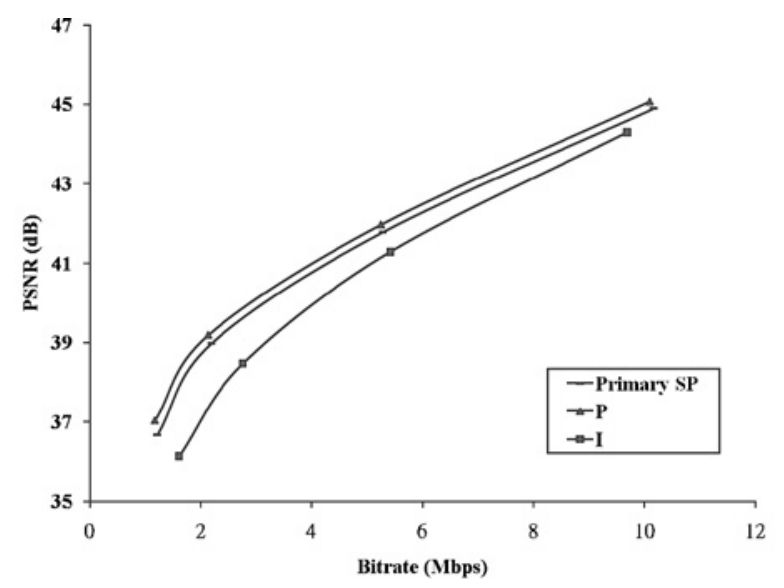

(a)

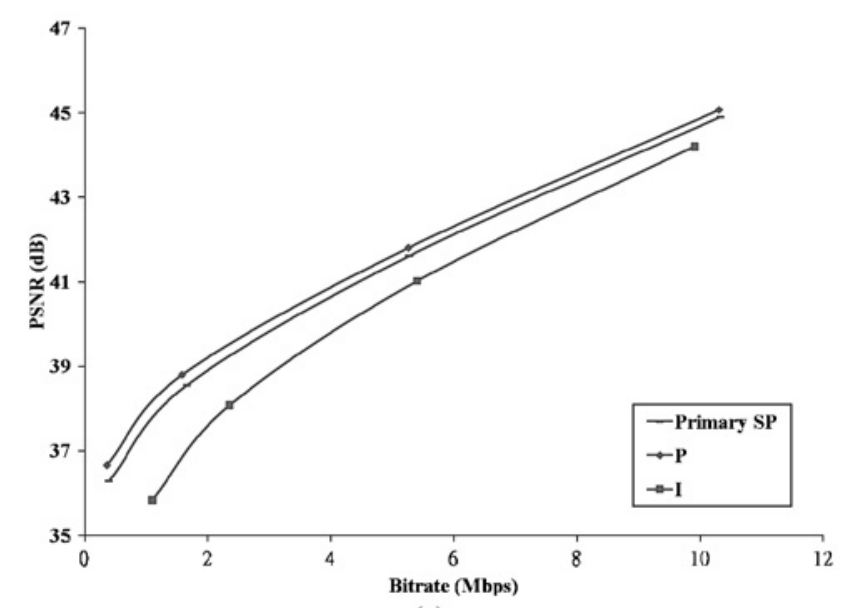

(c)

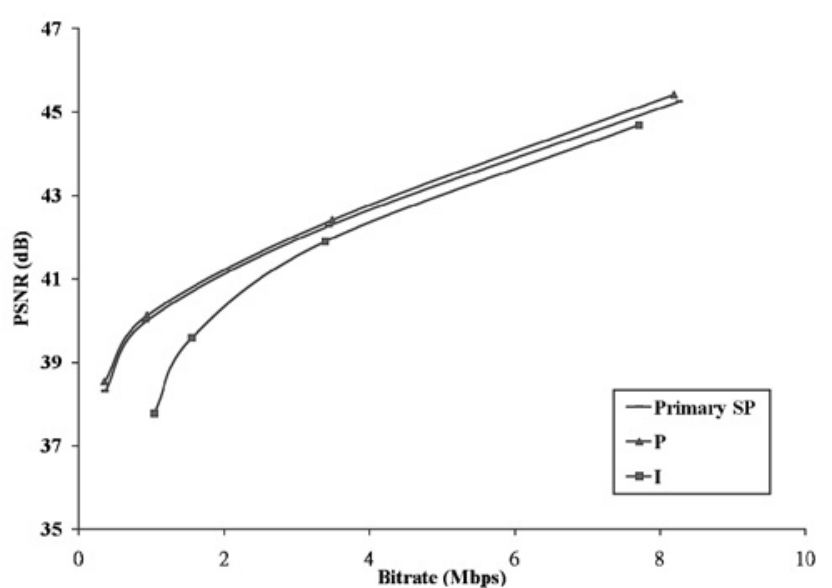

(b)

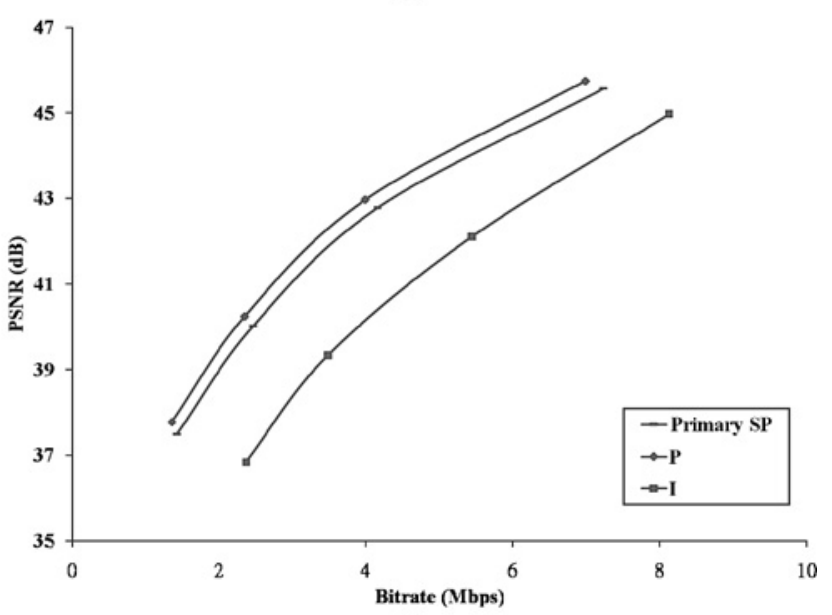

(d)

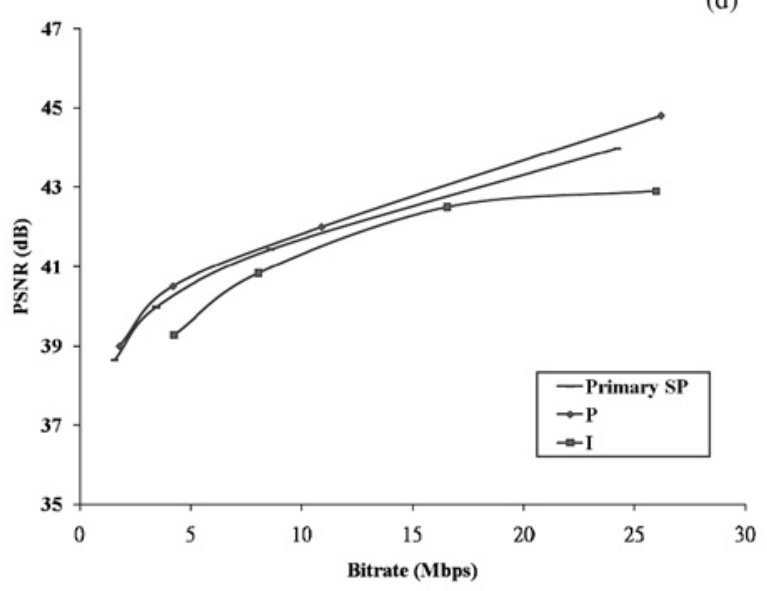

(e)

Fig. 17. Rate-distortion performance with periodic I-frame and SP-frame insertion for (a) Ballroom, (b) Exit, (c) Vassar, (d) Race1, and (e) Breakdancers.

frame 9, the user changes his/her viewpoint to 2 , the switching command sends from the client to the server through RTSP. The server cannot perform switching immediately, and waits for $S P_{12}$ at frame 12 . The server then sends this SP frame to the client which can now watch V2 from frame 12. $S P_{12}$ can perfectly reconstruct the primary SP-frame of V2 so that mismatch will not occur when switching is needed.

Before we analyze the storage requirement of secondary SP-frames encoded by our proposed scheme in the multiview video coding system, Fig. 17 gives the RD-performance when primary SP-frames are used instead of periodic I-frame insertion for the first view of Ballroom, Exit, Vassar, Race1, and Breakdancers. This figure also shows the results of the bitstreams encoded by all P-frames with the exception of the first frame, which is always an I-frame, for the reference. It can be observed in Fig. 17 that primary SP-frames have slightly lower coding efficiency than P-frames and significantly higher coding efficiency than I-frames, above $2 \mathrm{~dB}$. Therefore, periodic insertion of I-frames is not appropriate for viewpoint switching in MVV. 
TABLE IV

Average Bit-Counts of DifFerent SECONDARy SP-FrAmes For Exit By APPLYING THE SCHEME In [8] AND THE PROPOSED SCHEME

\begin{tabular}{|c|c|c|c|c|c|c|c|c|c|c|c|c|}
\hline$Q_{P}$ & \multicolumn{3}{|c|}{16} & \multicolumn{3}{|c|}{20} & \multicolumn{3}{|c|}{24} & \multicolumn{3}{|c|}{28} \\
\hline & $\begin{array}{l}\text { Scheme } \\
\text { in }[8] \\
(\mathrm{kb})\end{array}$ & $\begin{array}{l}\text { Proposed } \\
\text { Scheme } \\
(\mathrm{kb})\end{array}$ & $\Delta(\%)$ & $\begin{array}{l}\text { Scheme in } \\
{[8](\mathrm{kb})}\end{array}$ & $\begin{array}{l}\text { Proposed } \\
\text { Scheme } \\
(\mathrm{kb})\end{array}$ & $\Delta(\%)$ & $\begin{array}{l}\text { Scheme } \\
\text { in }[8] \\
(\mathrm{kb})\end{array}$ & $\begin{array}{l}\text { Proposed } \\
\text { Scheme } \\
(\mathrm{kb})\end{array}$ & $\Delta(\%)$ & $\begin{array}{l}\text { Scheme } \\
\text { in }[8] \\
(\mathrm{kb})\end{array}$ & $\begin{array}{l}\text { Proposed } \\
\text { Scheme } \\
(\mathrm{kb})\end{array}$ & $\Delta(\%)$ \\
\hline $\mathbf{0} \rightarrow \mathbf{1}$ & 821 & 804 & 2.0614 & 494 & 474 & 4.0056 & 247 & 240 & 2.8416 & 172 & 164 & 4.4850 \\
\hline $1 \rightarrow 2$ & 794 & 779 & 1.8081 & 482 & 467 & 3.0100 & 244 & 237 & 2.9492 & 164 & 161 & 1.3209 \\
\hline $2 \rightarrow 3$ & 789 & 775 & 1.7182 & 484 & 469 & 3.1425 & 252 & 245 & 2.9314 & 170 & 169 & 1.0919 \\
\hline $3 \rightarrow 4$ & 785 & 777 & 1.0732 & 501 & 485 & 3.1894 & 269 & 262 & 2.5766 & 180 & 178 & 1.1664 \\
\hline $4 \rightarrow 5$ & 797 & 777 & 2.4736 & 503 & 488 & 2.9856 & 272 & 264 & 3.0183 & 183 & 179 & 2.3214 \\
\hline $6 \rightarrow 7$ & 836 & 816 & 2.3772 & 575 & 550 & 4.3180 & 322 & 307 & 4.4248 & 215 & 208 & 3.1108 \\
\hline $\begin{array}{l}\text { Total } \\
\text { size }\end{array}$ & 5665 & 5532 & 2.3593 & 4013 & 3799 & 5.3177 & 2197 & 2079 & 5.3443 & 1501 & 1428 & 4.8524 \\
\hline
\end{tabular}

TABLE V

Average Bit-Counts of Different Secondary SP-Frames for Vassar By Applying the Scheme in [8] AND the Proposed Scheme

\begin{tabular}{|c|c|c|c|c|c|c|c|c|c|c|c|c|}
\hline$Q_{P}$ & \multicolumn{3}{|c|}{16} & \multicolumn{3}{|c|}{20} & \multicolumn{3}{|c|}{24} & \multicolumn{3}{|c|}{28} \\
\hline & $\begin{array}{l}\text { Scheme } \\
\text { in }[8] \\
(\mathrm{kb})\end{array}$ & $\begin{array}{l}\text { Proposed } \\
\text { Scheme } \\
(\mathrm{kb})\end{array}$ & $\Delta(\%)$ & $\begin{array}{l}\text { Scheme } \\
\text { in }[8] \\
(\mathrm{kb})\end{array}$ & $\begin{array}{l}\text { Proposed } \\
\text { Scheme } \\
(\mathrm{kb})\end{array}$ & $\Delta(\%)$ & $\begin{array}{l}\text { Scheme } \\
\text { in [8] } \\
(\mathrm{kb})\end{array}$ & $\begin{array}{l}\text { Proposed } \\
\text { Scheme } \\
(\mathrm{kb})\end{array}$ & $\Delta(\%)$ & $\begin{array}{l}\text { Scheme } \\
\text { in }[8] \\
(\mathrm{kb})\end{array}$ & $\begin{array}{l}\text { Proposed } \\
\text { Scheme } \\
(\mathrm{kb})\end{array}$ & $\Delta(\%)$ \\
\hline $\mathbf{0} \rightarrow \mathbf{1}$ & 934 & 930 & 0.4692 & 708 & 696 & 1.6166 & 402 & 392 & 2.5007 & 237 & 232 & 1.9908 \\
\hline $1 \rightarrow 2$ & 921 & 907 & 1.4547 & 678 & 668 & 1.5014 & 384 & 373 & 2.8764 & 233 & 228 & 2.1639 \\
\hline $2 \rightarrow 3$ & 933 & 925 & 0.7754 & 693 & 680 & 1.7555 & 395 & 383 & 2.8361 & 257 & 237 & 7.7057 \\
\hline $3 \rightarrow 4$ & 920 & 918 & 0.2328 & 690 & 682 & 1.0727 & 404 & 392 & 3.0209 & 243 & 240 & 1.0663 \\
\hline $4 \rightarrow 5$ & 902 & 897 & 0.6272 & 654 & 639 & 2.2440 & 388 & 372 & 4.2636 & 252 & 233 & 7.3849 \\
\hline $5 \rightarrow 6$ & 1354 & 1207 & 10.8053 & 999 & 869 & 13.0330 & 562 & 486 & 13.5240 & 372 & 321 & 13.6069 \\
\hline $6 \rightarrow 7$ & 990 & 975 & 1.5794 & 754 & 738 & 2.0984 & 464 & 447 & 3.6348 & 326329 & 294895 & 9.6325 \\
\hline $\begin{array}{l}\text { Total } \\
\text { size }\end{array}$ & 6954 & 6759 & 2.8003 & 5176 & 4974 & 3.9004 & 3000 & 2846 & 5.1333 & 327921 & 296386 & 9.6166 \\
\hline
\end{tabular}

TABLE VI

Average Bit-Counts of Different Secondary SP-Frames for Race1 by APPlying the Scheme in [8] AND the Proposed Scheme

\begin{tabular}{|c|c|c|c|c|c|c|c|c|c|c|c|c|}
\hline$Q_{P}$ & \multicolumn{3}{|c|}{16} & \multicolumn{3}{|c|}{20} & \multicolumn{3}{|c|}{24} & \multicolumn{3}{|c|}{28} \\
\hline & $\begin{array}{l}\text { Scheme } \\
\text { in }[8] \\
(\mathrm{kb})\end{array}$ & $\begin{array}{l}\text { Proposed } \\
\text { Scheme } \\
(\mathrm{kb})\end{array}$ & $\Delta(\%)$ & $\begin{array}{l}\text { Scheme } \\
\text { in }[8] \\
(\mathrm{kb})\end{array}$ & $\begin{array}{l}\text { Proposed } \\
\text { Scheme } \\
(\mathrm{kb})\end{array}$ & $\Delta(\%)$ & $\begin{array}{l}\text { Scheme } \\
\text { in }[8] \\
(\mathrm{kb})\end{array}$ & $\begin{array}{l}\text { Proposed } \\
\text { Scheme } \\
(\mathrm{kb})\end{array}$ & $\Delta(\%)$ & $\begin{array}{l}\text { Scheme } \\
\text { in }[8] \\
(\mathrm{kb})\end{array}$ & $\begin{array}{l}\text { Proposed } \\
\text { Scheme } \\
(\mathrm{kb})\end{array}$ & $\Delta(\%)$ \\
\hline $0 \rightarrow 1$ & 759 & 748 & 1.4538 & 538 & 524 & 2.5655 & 359 & 349 & 2.7901 & 255 & 246 & 3.6990 \\
\hline $1 \rightarrow 2$ & 765 & 748 & 2.2425 & 543 & 525 & 3.2461 & 356 & 349 & 1.9133 & 248 & 244 & 1.6617 \\
\hline $2 \rightarrow 3$ & 823 & 799 & 2.9566 & 600 & 565 & 5.7215 & 405 & 391 & 3.3618 & 276 & 264 & 4.4027 \\
\hline $3 \rightarrow 4$ & 826 & 796 & 3.6595 & 581 & 553 & 4.7872 & 391 & 368 & 5.7625 & 269 & 256 & 4.8631 \\
\hline $4 \rightarrow 5$ & 741 & 717 & 3.2204 & 512 & 491 & 4.1243 & 338 & 325 & 3.8777 & 239 & 231 & 3.2857 \\
\hline $5 \rightarrow 6$ & 1503 & 1324 & 11.9393 & 1105 & 960 & 13.0792 & 667 & 575 & 13.7502 & 436 & 372 & 14.6827 \\
\hline $6 \rightarrow 7$ & 818 & 802 & 1.9179 & 578 & 564 & 2.4605 & 376 & 367 & 2.4119 & 254 & 251 & 1.1549 \\
\hline $\begin{array}{l}\text { Total } \\
\text { size }\end{array}$ & 6235 & 5934 & 4.8400 & 4456 & 4183 & 6.1344 & 2891 & 2724 & 5.7697 & 1977 & 1864 & 5.7466 \\
\hline
\end{tabular}

In Fig. 16, there are seven secondary SP-frames to be stored in the server at each switching point in order to support switching among eight views. The benefits of adopting the proposed scheme for coding secondary SP-frames are to reduce the bits required for secondary SP-frames. As a consequence, the storage requirement of the video server can be reduced. Tables III-VII summarize the average sizes of secondary SPframes with different $Q_{P}$ for the test sequences Ballroom, Exit, Vassar, Racel, and Breakdancers, respectively. The sizes of different secondary SP-frames between two views such as view 1 to view $2(1 \rightarrow 2)$, view 2 to view $3(2 \rightarrow 3)$, etc., are also shown. Each row contains the average bit-counts of encoding SP-frames using the pixel-domain and QDCT-domain motion estimation techniques that need to be stored in the server, and the percentage reduction of our proposed scheme over the scheme in [8] at different switching viewpoints. The last row of each table shows the total storage requirement of secondary SP-frames in the server by using different schemes. 
TABLE VII

Average Bit-Counts of Different Secondary SP-Frames For Breakdancers By APPLying the Scheme IN [8] AND THE PROPOSED SCHEME

\begin{tabular}{|c|c|c|c|c|c|c|c|c|c|c|c|c|}
\hline$Q_{P}$ & \multicolumn{3}{|c|}{16} & \multicolumn{3}{|c|}{20} & \multicolumn{3}{|c|}{24} & \multicolumn{3}{|c|}{28} \\
\hline & $\begin{array}{l}\text { Scheme } \\
\text { in }[8] \\
(\mathrm{kb})\end{array}$ & $\begin{array}{l}\text { Proposed } \\
\text { Scheme } \\
(\mathrm{kb})\end{array}$ & $\Delta(\%)$ & $\begin{array}{l}\text { Scheme } \\
\text { in }[8] \\
(\mathrm{kb})\end{array}$ & $\begin{array}{l}\text { Proposed } \\
\text { Scheme } \\
\text { (kb) }\end{array}$ & $\Delta(\%)$ & $\begin{array}{l}\text { Scheme } \\
\text { in }[8] \\
(\mathrm{kb})\end{array}$ & $\begin{array}{l}\text { Proposed } \\
\text { Scheme } \\
(\mathrm{kb})\end{array}$ & $\Delta(\%)$ & $\begin{array}{l}\text { Scheme } \\
\text { in }[8] \\
(\mathrm{kb})\end{array}$ & $\begin{array}{l}\text { Proposed } \\
\text { Scheme } \\
(\mathrm{kb})\end{array}$ & $\Delta(\%)$ \\
\hline $0 \rightarrow 1$ & 2034 & 1986 & 2.3442 & 1098 & 1040 & 5.3135 & 559 & 527 & 5.7457 & 322 & 311 & 3.5559 \\
\hline $1 \rightarrow 2$ & 2004 & 1967 & 1.8627 & 1056 & 1024 & 3.0016 & 546 & 508 & 6.8279 & 291 & 284 & 2.2984 \\
\hline $2 \rightarrow 3$ & 1980 & 1937 & 2.1576 & 1036 & 990 & 4.4812 & 523 & 487 & 6.9169 & 283 & 279 & 1.5895 \\
\hline $3 \rightarrow 4$ & 1938 & 1885 & 2.7342 & 1022 & 949 & 7.1691 & 517 & 491 & 5.0222 & 283 & 279 & 1.5011 \\
\hline $4 \rightarrow 5$ & 1988 & 1936 & 2.6376 & 1002 & 978 & 2.3458 & 533 & 502 & 5.8334 & 294 & 287 & 2.3450 \\
\hline $5 \rightarrow 6$ & 1990 & 1924 & 3.2814 & 1053 & 980 & 6.9283 & 492 & 470 & 4.3232 & 288 & 279 & 3.0599 \\
\hline $6 \rightarrow 7$ & 2034 & 1991 & 2.1338 & 1118 & 1042 & 6.7325 & 537 & 522 & 2.8077 & 327 & 305 & 6.6016 \\
\hline $\begin{array}{l}\text { Total } \\
\text { size }\end{array}$ & 13968 & 13626 & 2.4474 & 7385 & 7004 & 5.1653 & 3707 & 3508 & 5.3673 & 2088 & 2024 & 3.0735 \\
\hline
\end{tabular}

From these tables, it can easily be observed that the required storage in the server of the proposed scheme is much fewer than that of the scheme in [8], which provides up to $3 \%, 5 \%$, $10 \%, 6 \%$, and 5\% savings in Ballroom, Exit, Vassar, Race1, and Breakdancers, respectively. Again, the merit of proposed scheme is mainly due to performing motion estimation in the QDCT domain, which obtains more desirable motion vectors for entropy coding of secondary SP-frames. Moreover, in a multiview system, switching viewpoints may be necessary to come across all the in-between views. Implementing SPframes can achieve drift-free and low-delay switching among views. With our proposed scheme, the total sizes of switching SP-frames can then be reduced and this is also favorable to the bandwidth requirements of multiview streaming.

\section{CONCLUSION}

In this paper, an efficient scheme for coding H.264 secondary SP-frames has been proposed. We found that the use of conventional pixel-domain motion estimation is not appropriate for a secondary SP-frame encoder in multiview video and single-view video with complex motion, which incurs considerable size of secondary SP-frames. To alleviate this, we have incorporated the QDCT-domain motion estimation technique in the encoding process of secondary SP-frames. The new technique utilizes an appropriate cost function, which is operated in the QDCT domain, for motion estimation in coding of secondary SP-frames. Based on the new metrics, experimental results showed that the proposed scheme significantly reduced the size of H.264 secondary SP-frames. Besides, the proposed technique does not affect the coding efficiency of primary SP-frames. Moreover, the proposed scheme is particularly useful to low-delay viewpoint switching in the multiview video system in which users will be more interested in switching their views during an exciting event. An exciting event is always in the scene consisting of complex motion and inter-view disparity, and the correlation between two views becomes lower. In this scenario, the proposed scheme is beneficial to the entropy coding of secondary SP-frames.

Nevertheless, some issues still remain to be investigated when the proposed QDCT-domain technique is adopted in secondary SP-frame coding. For example, the gains of the proposed QDCT-domain technique over the pixel-domain motion estimation techniques vary quite widely over different $Q_{P}$ and view pairs. One of the reasons is that the Lagrangian multiplier for motion estimation $\left(\lambda_{\text {motion }}\right)$ defined in (1) depends on $Q_{P}$, and it is designed for the pixel-domain technique. But, the proposed QDCT-ME is operated in the quantized DCT domain. A careful selection of $\lambda_{\text {motion }}$ based on the mechanism of secondary SP-frame coding, SAQTD and energy compensation factor $k$ should further improve the performance of our proposed QDCT-domain technique. Last but not least, one of our future works could also focus on techniques to reduce the computational complexity of the proposed QDCTdomain scheme. For instance, a selection mechanism based on the interview correlation should be adopted to choose between the use of pixel-domain and quantized transformdomain techniques. This idea can save the complexity due to the QDCT-domain technique, and this could be a point for our further investigation.

\section{REFERENCES}

[1] Advanced Video Coding for Generic Audiovisual Services, ITU-T Rec. H.264 and ISO/IEC 14496-10:2005 Standards, Mar. 2005.

[2] G. Sullivan and T. Wiegand, "Video compression: From concepts to the H.264/AVC video coding standard," Proc. IEEE, vol. 93, no. 1, pp. 18-31, Jan. 2005.

[3] D. Marpe, T. Wiegand, and G. J. Sullivan, "The H.264/MPEG-4 advanced video coding standard and its applications," IEEE Commun. Mag., vol. 44, no. 8, pp. 134-143, Aug. 2006.

[4] R. Kurceren and M. Karczewicz, "Synchronization-predictive coding for video compression: The SP frames design for JVT/H.26L," in Proc. IEEE Int. Conf. Image Process., vol 2. Sep. 2002, pp. 497-500.

[5] M. Karczewicz and R. Kurceren, "The SP and SI-frames design for H.264/AVC," IEEE Trans. Circuits Syst. Video Technol., vol. 13, no. 7, pp. 637-644, Jul. 2003.

[6] C.-P. Chang and C.-W. Lin, "RD optimized quantization of H.264 SPframes for bitstream switching under storage constraints," in Proc. IEEE Int. Symp. Circuits Syst, vol. 2. May 2005, pp. 1242-1245.

[7] E. Setton and B. Girod, "Rate-distortion analysis and streaming of SP and SI frames," IEEE Trans. Circuits Syst. Video Technol., vol. 16, no. 6, pp. 733-743, Jun. 2006.

[8] W. T. Tan and B. Shen, "Methods to improve coding efficiency of SP frames," in Proc. IEEE Int. Conf. Image Process., Atlanta, GA, Oct. 2006, pp. 1361-1364.

[9] A. Smolic and D. M. Cutchen, "3DAV exploration of video-based rendering technology in MPEG," IEEE Trans. Circuits Syst. Video Technol., vol. 14, no. 9, pp. 348-356, Mar. 2004. 
[10] Requirements on Multi-View Video Coding v.5, document N7539.doc, ISO/IEC/JTC1/SC29/WG11, Nice, France, Oct. 2005.

[11] Report of the Subjective Quality Evaluation for MVC Call for Evidence, document N6999.doc, ISO/IEC/JTC1/SC29/WG11, Hong Kong, China, 2005.

[12] Call for Proposal on Multi-View Video Coding, document N7327.doc, ISO/IEC/JTC1/SC29/WG11, Poznan, Poland, 2005.

[13] P. Merkle, A. Smolic, K. Muller, and T. Wiegand, "Efficient prediction structure for multiview video coding," IEEE Trans. Circuits Syst. Video Technol., vol. 17, no. 11, pp. 1461-1473, Nov. 2007.

[14] M. Flierl, A. Mavlankar, and B. Girod, "Motion and disparity compensated coding for multi-view video," IEEE Trans. Circuits Syst. Video Technol., vol. 17, no. 11, pp. 1474-1484, Nov. 2007.

[15] X. Guo, Y. Lu, W. Gao, and Q. Huang, "Viewpoint switching in multiview video streaming," in Proc. IEEE Int. Symp. Circuits Syst., vol. 4. Kobe, Japan, May 2005, pp. 3417-3474.

[16] X. Guo, Y. Lu, F. Wu, W. Gao, and S. Li, "Free viewpoint switching in multi-view video streaming using Wyner-Ziv video coding," in Proc. Int. Soc. Opt. Engineers Visual Commun. Image Process., vol. 6077. San Jose, CA, Jan. 2006, pp. 60770U1-8.

[17] J. G. Lou, H. Cai, and J. Li, "A real-time interactive multi-view video system," in Proc. 13th Annu. Assoc. Comput. Machinery Int. Conf. Multimedia, Nov. 2005, Singapore, pp. 161-170.

[18] L. Zuo, J. G. Lou, H. Cai, and J. Li, "Multicast of real-time multiview video," in Proc. IEEE Int. Conf. Multimedia Expo, Jul. 2006, pp. $1225-1228$.

[19] EyeVision Project [Online]. Available: http://www.ri.cmu.edu/projects/ project_449.html

[20] K. Suhring (2006). H.264 Reference Software JM11.0 [Online]. Available: http://iphome.hhi.de/suehring/tml

[21] U.-V. Koc and K. J. R. Liu, "DCT-based motion estimation," IEEE Trans. Image Process., vol. 7, no. 7, pp. 948-965, Jul. 1998

[22] M. Li, M. Biswas, S. Kumar, N. Truong, "DCT-based phase correlation motion estimation," in Proc. Int. Conf. Image Process., vol. 1. Oct. 2004, pp. 445-448.

[23] Multiview Video Test Sequences from MERL, document MPEG05/ m12077.doc, ISO/IEC/JTC1/SC29/WG11, Apr. 2005.

[24] Call for Proposals on Multi-View Video Coding, document N7327.doc, ISO/IEC/JTC1/SC29/WG11, Poznan, Poland, Jul. 2005.

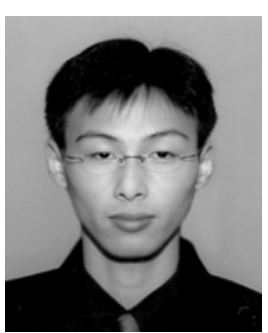

Ki-Kit Lai received the B.Eng. (Honors) and M.Eng. degrees in electronic and information engineering from The Hong Kong Polytechnic University, Hung Hom, Kowloon, Hong Kong, in 2006. He is currently pursuing the Ph.D. degree in electronic and information engineering at the Center for Signal Processing, Department of Electronic and Information Engineering, The Hong Kong Polytechnic University, under the supervision of Dr. Y. L. Chan and Prof. W. C. Siu.

His research interests include image and video technology, video compression, and multiview video coding.

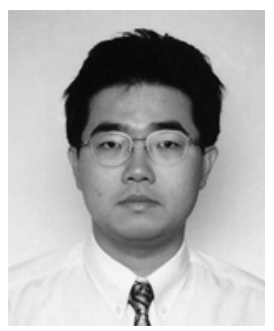

Yui-Lam Chan (S'94-A'97-M'00) received the B.Eng. degree (First Class Honors), and the Ph.D. degree both in electronic and information engineering from The Hong Kong Polytechnic University, Hung Hom, Kowloon, Hong Kong, in 1993 and 1997, respectively.

He joined The Hong Kong Polytechnic University in 1997, and is currently an Assistant Professor with the Center for Signal Processing, Department of Electronic and Information Engineering. He has published over 60 research papers in various international journals and conferences. His research and technical interests include multimedia technologies, signal processing, image and video compression, video streaming, video transcoding, video conferencing, digital TV/highdefinition TV, multiview video coding, future video coding standards, errorresilient coding, and digital video cassette recording.

Dr. Chan received both the Faculty Merit Award in Teaching (Team) and the Faculty of Engineering Research Grant Achievement Award, in 2005. During his studies, he was the recipient of more than ten prizes, scholarships, and fellowships for his outstanding academic achievement, including the
Champion in Varsity Competition in Electronic Design, the Sir Edward Youde Memorial Fellowship, and the Croucher Foundation Scholarships. He is also actively involved in professional activities. He serves as a Reviewer and Session Chairman for many international journals and conferences. He was the Registration Chair of the 2004 International Symposium on Intelligent Multimedia, Video, and Speech Processing held in Hong Kong between October 20 and 22, 2004. He is the Secretary of the coming 2010 IEEE International Conference on Image Processing.

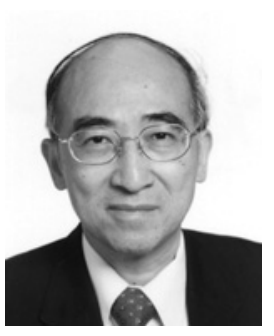

Wan-Chi Siu (S'77-M'77-SM'90) received an Associateship from The Hong Kong Polytechnic University, Hung Hom, Kowloon, Hong Kong, and the M.Phil. degree from the Chinese University of Hong Kong, Sha Tin, Hong Kong, both in electronic engineering, in 1975 and 1977, respectively, and the Ph.D. degree in electronic engineering from the Imperial College of Science, Technology and Medicine, London, U.K., in October 1984.

He was with the Chinese University of Hong Kong as a Tutor and later as an Engineer between 1975 and 1980. He then joined The Hong Kong Polytechnic University as a Lecturer in 1980. He was promoted to the Senior Lecturer, Principal Lecturer, and Reader in 1985, 1987, and 1990, respectively, and has been the Chair Professor of the Department of Electronic and Information Engineering since 1992. He is currently the Director of the Center for Multimedia Signal Processing, Department of Electronic and Information Engineering, The Hong Kong Polytechnic University. He is an Editor of the book Multimedia Information Retrieval and Management (Berlin, Heidelberg: Springer, 2003). His works on fast computational algorithms such as discrete cosine transform and motion estimation algorithms have been well accepted by academic peers, with good citation records, and a number of them are now being used in high tech industrial applications. His research interests include transforms, image processing, and the computational aspects of pattern recognition and wavelets.

Prof. Siu is an expert and a popular speaker in digital signal processing, specializing in fast algorithms and video coding. He frequently makes open presentations and has published 380 research papers, over 150 of which appeared in various international journals, such as IEEE TRANSACTIONS. He has been the Keynote and Invited Speaker, and the Key Organizer of many world-class international conferences, such as the IEEE International Conference on Neural Networks and Signal Processing in 2008 (Keynote Speaker, China), the IEEE International Conference on Information and Communication Systems in 2007 (Invited Speaker, Singapore), the IEEE Continuous-Phase Modulation in 2002 (Keynote Speaker, Taiwan), the IEEE Conference on Multimedia Signal Processing in 2008 (General Co-Chair, Australia), and some IEEE society sponsored flagship conferences, including the International Symposium on Circuits and Systems in 1997 (Technical Program Chair), the International Conference on Acoustics, Speech, and Signal Processing in 2003 (General Chair), and the International Conference on Image Processing in 2010 (General Chair). He has been and was the Guest Editor, the Associate Editor, and the Member of the Editorial Board of a number of journals, including the IEEE TRANSACTIONS ON CIRCUITS AND SYSTEMS, PART II, PATTERN RECOGNITION, the Journal of Very Large Scale Integration Signal Processing Systems for Signal, Image, Video Technology, and the EURASIP Journal on Applied Signal Processing, in addition to other journals. In 1994, he chaired the First Engineering and Information Technology Panel of the Research Assessment Exercise to assess the research quality of 19 cost centers (departments) from all universities in Hong Kong. 